\title{
Palladium Catalyzed Tandem Ring Opening- Ring Closing Reaction of Diazabicyclic Alkenes: A Facile One Pot Strategy for Cyclopentannulation of Heterocycles
}

\author{
Jubi John, ${ }^{a}$ Indu U., ${ }^{a}$ Eringathodi Suresh ${ }^{\mathrm{b}}$ and K. V. Radhakrishnan* ${ }^{\mathrm{a}}$ \\ ${ }^{a}$ Organic Chemistry Section, National Institute for Interdisciplinary Science and \\ Technology (CSIR), Trivandrum 695 019, India, \\ ${ }^{b}$ Central Salt and Marine Chemicals Research Institute, Bhavnagar 364 002, India. \\ E-mail: radhupreethi@ rediffmail.com
}

\section{Table of Contents}

General Methods

Experimental Procedures

Optimization Studies

Characterization of the Products

ORTEP drawing of $\mathbf{3 c}$

${ }^{1} \mathrm{H}$ NMR \& ${ }^{13} \mathrm{C}$ NMR Spectra
S2

S2-S4

S4-S6

S7-S14

S15-S16

S17-S46 


\section{General Methods}

All the chemicals were of the best grade commercially available and are used without further purification. All the solvents were purified according to standard procedure; dry solvents were obtained according to the literature methods and stored over molecular sieves. Analytical thin layer chromatography was performed on glass plates coated with silica gel containing calcium sulfate binder. Gravity column chromatography was performed using 60-120 mesh silica gel or neutral alumina and mixtures of hexane-ethyl acetate were used for elution.

Melting points was determined on a Buchi melting point apparatus and is uncorrected. Proton nuclear magnetic resonance spectra $\left({ }^{1} \mathrm{H}\right.$ NMR) were recorded on a Bruker Avance DPX 300 and Bruker AMX 500 spectrophotometer $\left(\mathrm{CDCl}_{3}\right.$ as solvent). Chemical shifts for ${ }^{1} \mathrm{H}$ NMR spectra are reported as $\delta$ in units of parts per million (ppm) downfield from $\mathrm{SiMe}_{4}(\delta 0.0)$ and relative to the signal of chloroform-d $(\delta 7.25$, singlet). Multiplicities were given as: s (singlet); d (doublet); t (triplet); brs (broad singlet); m (multiplet). Coupling constants are reported as $J$ value in $\mathrm{Hz}$.

Carbon nuclear magnetic resonance spectra $\left({ }^{13} \mathrm{C} \mathrm{NMR}\right)$ are reported as $\delta$ in units of parts per million (ppm) downfield from $\mathrm{SiMe}_{4}(\delta 0.0)$ and relative to the signal of chloroform-d ( $\delta 77.03$, triplet).

Mass spectra were recorded under EI/HRMS and FAB/LRMS at 5000 resolution using JMS600H (Jeol) mass spectrometer. IR spectra were recorded on Bomem MB series FT-IR spectrometer.

\section{Typical Procedure for the preparation of 2,3-Diazabicyclo [2.2.1] hept-5-ene 1(a-d).}

Diethyl azodicarboxylate [0.90 mL, $5.7418 \mathrm{mmol}]$ was dissolved in ether and cooled to $0{ }^{\circ} \mathrm{C}$. Cyclopentadiene $[0.77 \mathrm{~mL}, 11.4837 \mathrm{mmol}]$ was added to it and the reaction mixture was stirred for 12 hours. Excess cyclopentadiene was added to it if yellow color persists in the reaction mixture. Completion of the reaction was monitored by TLC and ether was evaporated off. The crude sample on purification by column chromatography (silica gel 100-200 mesh, 15\% EtOAc-Hexane) afforded the product as a light yellow viscous liquid in $100 \%$ yield $(1.3 \mathrm{~g})$. 
General experimental procedure for the reaction of bicyclic hydrazine with 2iodophenol:

Bicyclic hydrazine (1.5 equiv.), 2-iodophenol (1 equiv.), $\mathrm{Bu}_{4} \mathrm{NCl}$ (1 equiv.), $\mathrm{K}_{2} \mathrm{CO}_{3}$ (2 equiv.) and $[\mathrm{Pd}(\text { allyl }) \mathrm{Cl}]_{2}(5 \mathrm{~mol} \%)$ were taken in a Wheaton reactor. The mixture was dissolved in $[\mathrm{bmim}]\left[\mathrm{PF}_{6}\right](2 \mathrm{~mL})$ and stirred at $80{ }^{\circ} \mathrm{C}$ for 8 hours under argon atmosphere. After the completion of the reaction, the reaction mixture was extracted with ether, dried over $\mathrm{Na}_{2} \mathrm{SO}_{4}$. The solvent was evaporated in vacuo and the residue on silica gel (60-120 mesh) column chromatography using 30\% ethyl acetate in hexane afforded the product in good yield.

General experimental procedure for the reaction of bicyclic hydrazine with 2iodoaniline:

Bicyclic hydrazine (1.5 equiv.), 2-iodoaniline (1 equiv.), $\mathrm{LiCl}$ (1 equiv.), $\mathrm{K}_{2} \mathrm{CO}_{3}(2$ equiv. $)$ and $\mathrm{Pd}(\mathrm{OAc})_{2}(5 \mathrm{~mol} \%)$ were taken in a Wheaton reactor. The mixture was dissolved in Toluene $(2 \mathrm{~mL})$ and stirred at $100{ }^{\circ} \mathrm{C}$ for 36 hours under argon atmosphere. After the completion of the reaction, the solvent was evaporated in vacuo and the residue on neutral alumina column chromatography using mixtures of hexane and ethyl acetate in hexane afforded the product in good yield.

General experimental procedure for the preparation of 3,4- disubstituted cyclopentene from bicyclic hydrazine and 2-iodophenol:

Bicyclic hydrazine (1.5 equiv.), 2-iodophenol (1 equiv.), $\mathrm{PPh}_{3}(10 \mathrm{~mol} \%), \mathrm{K}_{2} \mathrm{CO}_{3}$ (2 equiv.) and $[\mathrm{Pd}(\text { allyl }) \mathrm{Cl}]_{2}(5 \mathrm{~mol} \%)$ were taken in a Wheaton reactor. The mixture was dissolved in $\mathrm{CH}_{3} \mathrm{CN}(2 \mathrm{~mL})$ and stirred at $60{ }^{\circ} \mathrm{C}$ for 16 hours under argon atmosphere. After the completion of the reaction, the solvent was evaporated in vacuo and the residue on silica gel (60-120 mesh) column chromatography using $20 \%$ ethyl acetate in hexane afforded the product in good yield.

General experimental procedure for the preparation of 3,4- disubstituted cyclopentene from bicyclic hydrazine and 2-iodoaniline:

Bicyclic hydrazine (1.5 equiv.), 2-iodoaniline (1 equiv.), $\mathrm{PPh}_{3}(10 \mathrm{~mol} \%), \mathrm{K}_{2} \mathrm{CO}_{3}$ (2 equiv.) and $\mathrm{Pd}(\mathrm{OAc})_{2}(5 \mathrm{~mol} \%)$ were taken in a Wheaton reactor. The mixture was dissolved in $\mathrm{CH}_{3} \mathrm{CN}(2 \mathrm{~mL})$ and stirred at $60{ }^{\circ} \mathrm{C}$ for 24 hours under argon atmosphere. After the completion of the reaction, the solvent was evaporated in vacuo and the residue 
on neutral alumina column chromatography using $20 \%$ ethyl acetate in hexane afforded the product in good yield.

\section{Optimization Studies for Palladium- catalyzed reaction of Azabicyclic Olefins with}

\section{2-Iodophenol (Table 1)}

\begin{tabular}{|c|c|c|c|c|c|}
\hline Entry & Catalyst & Solvent & Base & Additive & Yield $(\%)^{a}$ \\
\hline 1 & $\mathrm{Pd}(\mathrm{OAc})_{2}$ & DMF & $\mathrm{K}_{2} \mathrm{CO}_{3}$ & $\mathrm{Bu}_{4} \mathrm{NCl}$ & 63 \\
\hline 2 & $\mathrm{Pd}(\mathrm{OAc})_{2}$ & " & " & $\mathrm{Bu}_{4} \mathrm{NBr}$ & No Reaction \\
\hline 3 & $\mathrm{Pd}(\mathrm{OAc})_{2}$ & " & $"$ & $\mathrm{Bu}_{4} \mathrm{NI}$ & No Reaction \\
\hline 4 & $\mathrm{Pd}(\mathrm{OAc})_{2}$ & $"$ & $"$ & - & No Reaction \\
\hline 5 & $\mathrm{Pd}(\mathrm{OAc})_{2}$ & " & $\mathrm{Na}_{2} \mathrm{CO}_{3}$ & $\mathrm{Bu}_{4} \mathrm{NCl}$ & 22 \\
\hline 6 & $\mathrm{Pd}(\mathrm{OAc})_{2}$ & $"$ & $\mathrm{Cs}_{2} \mathrm{CO}_{3}$ & " & Trace \\
\hline 7 & $\operatorname{Pd}\left[(\text { allyl) } \mathrm{Cl}]_{2}\right.$ & $"$ & $\mathrm{~K}_{2} \mathrm{CO}_{3}$ & $"$ & 91 \\
\hline 8 & $\mathrm{PdCl}_{2}$ & " & $"$ & $"$ & 32 \\
\hline 9 & $\mathrm{Pd}\left(\mathrm{PPh}_{3}\right)_{4}$ & $"$ & $"$ & $"$ & 33 \\
\hline 10 & {$\left[\mathrm{Pd}(\text { allyl) } \mathrm{Cl}]_{2}\right.$} & $\mathrm{CH}_{3} \mathrm{CN}$ & " & " & 93 \\
\hline 11 & {$[\mathrm{Pd}(\mathrm{allyl}) \mathrm{Cl}]_{2}$} & THF & " & " & 37 \\
\hline 12 & {$[\mathrm{Pd}(\mathrm{allyl}) \mathrm{Cl}]_{2}$} & Toluene & " & " & 26 \\
\hline 13 & {$\left[\mathrm{Pd}(\text { allyl) } \mathrm{Cl}]_{2}\right.$} & {$[\mathrm{bmim}]\left[\mathrm{PF}_{6}\right]$} & $"$ & $"$ & $94^{\mathrm{b}}$ \\
\hline 14 & {$\left[\mathrm{Pd}(\text { allyl) } \mathrm{Cl}]_{2}\right.$} & {$[\mathrm{bmim}]\left[\mathrm{PF}_{6}\right]$} & " & $"$ & Trace $^{c}$ \\
\hline
\end{tabular}

Reaction Conditions: alkene ( 1.5 equiv.), 2- iodophenol ( 1 equ iv.), catalyst (5 mol\%), base (2 equiv.), add itive (1 equiv.), solvent $(2 \mathrm{~mL}), 80^{\circ} \mathrm{C}, 24 \mathrm{~h}$.

${ }^{a}$ Isolated yield, ${ }^{b}$ Reaction time $8 \mathrm{~h},{ }^{\mathrm{c}} \mathrm{rt}, 2$ days.

Table 1 describes our efforts towards optimizing various reaction parameters. Among the additives that were examined $\mathrm{Bu}_{4} \mathrm{NBr}$ and $\mathrm{Bu}_{4} \mathrm{NI}$ were found to be ineffective (Table 1, entry 2 and 3). However the reaction was not working in the absence of $\mathrm{Bu}_{4} \mathrm{NCl}$ (Table 1, entry 4). We went on optimizing other parameters such as base, catalyst and solvent. A base screen revealed that $\mathrm{K}_{2} \mathrm{CO}_{3}$ was more effective than $\mathrm{Na}_{2} \mathrm{CO}_{3}$ and $\mathrm{Cs}_{2} \mathrm{CO}_{3}$ (Table 1, entry 1, 5 and 6). Various catalysts such as $\mathrm{Pd}(\mathrm{OAc})_{2},[\mathrm{Pd}(\text { allyl }) \mathrm{Cl}]_{2}, \mathrm{PdCl}_{2}$, $\operatorname{Pd}\left(\mathrm{PPh}_{3}\right)_{4}$ were screened from which $[\mathrm{Pd}(\text { allyl }) \mathrm{Cl}]_{2}$ gave the highest yield (Table 1, entry 1, 7-9). We then turned our attention to solvent effects. An increase in reaction efficiency 
was observed with DMF, $\mathrm{CH}_{3} \mathrm{CN}$ and ionic liquid [bmim] $\left[\mathrm{PF}_{6}\right]$ (Table 1, entry 7, 10 and 13). The rate of reaction was found to be accelerated in $[\mathrm{bmim}]\left[\mathrm{PF}_{6}\right]$ as observed earlier in our studies with bicyclic hydrazines. ${ }^{6 a, b}$ Lowering the temperature resulted in sluggish reaction (Table 1, entry 14$)$.

Optimization Studies for Palladium- catalyzed reaction of Azabicyclic Olefins with 2-Iodoaniline (Table 2)

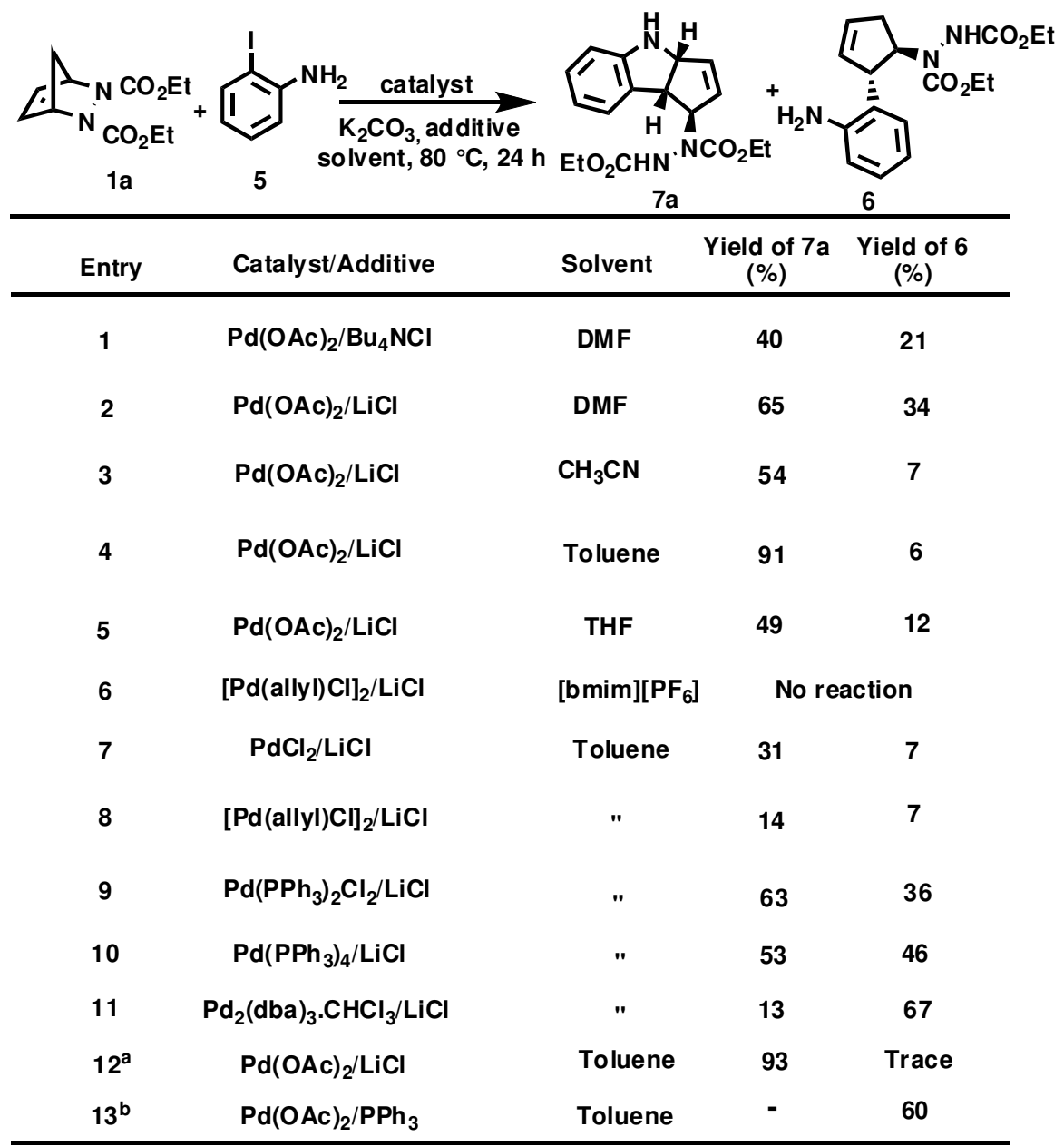

Reaction Conditions: alkene ( 1.5 equiv.), 2 - iodoaniline ( 1 equiv.), catalyst (5 mol\%), $\mathrm{K}_{2} \mathrm{CO}_{3}$ (2 equiv.), $\mathrm{LiCl}$ ( 1 equiv.), solvent $(2 \mathrm{~mL}), 80{ }^{\circ} \mathrm{C}, 24 \mathrm{~h}$. a $100{ }^{\circ} \mathrm{C}, 36 \mathrm{~h} .{ }^{\mathrm{b}} 60^{\circ} \mathrm{C}$

Table 2 describes our efforts towards optimizing various reaction parameters. Among the additives scanned $\mathrm{LiCl}$ was found to be more effective (Table 2, entry 1 and 2). From the solvents tested, toluene was found to be the best for the formation of cyclopentannulated product (Table 2, entry 4). The reaction did not work in ionic liquid $[$ bmim $]\left[\mathrm{PF}_{6}\right]$ (Table 2, entry 6). The catalysts screened were $\mathrm{Pd}(\mathrm{OAc})_{2},[\mathrm{Pd}(\text { allyl }) \mathrm{Cl}]_{2}$, $\mathrm{PdCl}_{2}, \mathrm{PdCl}_{2}\left(\mathrm{PPh}_{3}\right)_{2}, \mathrm{Pd}_{2} \mathrm{dba}_{3} \cdot \mathrm{CHCl}_{3}$ and $\mathrm{Pd}\left(\mathrm{PPh}_{3}\right)_{4}$, from which $\mathrm{Pd}(\mathrm{OAc})_{2}$ gave the 
highest yield (Table 2, entry 4, 7- 11). An increase in the reaction temperature and time lead to the decrease in the formation of 3,4-disubstituted cyclopentene (Table 2, entry 12). When the reaction was repeated by replacing the chloride salt by $\mathrm{PPh}_{3}$, the exclusive formation of 3,4-disubstituted cyclopentene 6 was observed (Table 2, entry 13). 


\section{Characterization of the products:}

Diethyl 1-((1R,3aR,8bS)-3a,8b-dihydro-1H-benzo[b]cyclopenta[d]furan-1yl)hydrazine-1,2-dicarboxylate (3a).

Yield : $94 \%$ as colorless viscous liquid.

$\mathrm{R}_{\mathrm{f}}: 0.42$ (hexane/ethyl acetate $=6: 4$ ).

IR (KBr) $v_{\max }: 3396,2987,2927,2854,1749,1710,1595$,

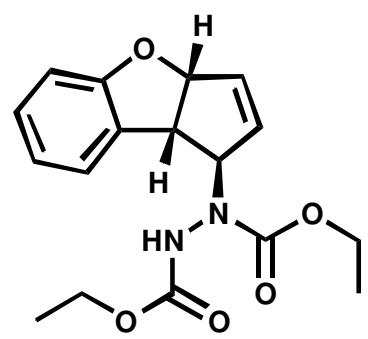
$1481,1307,1253,1132,914,813 \mathrm{~cm}^{-1}$.

${ }^{1} \mathrm{H}$ NMR $\left(300 \mathrm{MHz}, \mathrm{CDCl}_{3}\right): \delta 7.36$ (brs, $\left.1 \mathrm{H}\right), 7.12(\mathrm{t}, 1 \mathrm{H}$, $J=7.50 \mathrm{~Hz}), 6.87(\mathrm{t}, 1 \mathrm{H}, J=7.40 \mathrm{~Hz}), 6.74-6.71(\mathrm{~d}, 1 \mathrm{H}, J$ $=7.95 \mathrm{~Hz}), 6.29(\mathrm{~s}, 1 \mathrm{H}), 6.09(\mathrm{~m}, 1 \mathrm{H}), 5.86-5.81(\mathrm{~m}, 2 \mathrm{H})$, $5.34(\mathrm{~s}, 1 \mathrm{H}), 4.27-4.16(\mathrm{~m}, 4 \mathrm{H}), 4.11(\mathrm{~s}, 1 \mathrm{H}), 1.34-1.26(\mathrm{~m}$, $6 \mathrm{H})$.

${ }^{13} \mathrm{C}$ NMR $\left(125 \mathrm{MHz}, \mathrm{CDCl}_{3}\right): \delta 158.1,156.6,155.6,135.3$, $132.3,128.9,128.2,125.3,120.8,110.1,90.8,71.8,62.8$, $62.4,50.2,14.6,14.4$.

HRMS (EI): Calcd for $\mathrm{C}_{17} \mathrm{H}_{20} \mathrm{~N}_{2} \mathrm{O}_{5}, \mathrm{M}^{+}$: 332.1372; Found: 332.1383 .

\section{Diisopropyl 1-((1R,3aR,8bS)-3a,8b-dihydro-1H-benzo[b]cyclopenta[d]furan-1-} yl)hydrazine-1,2-dicarboxylate (3b).

Yield : $88 \%$ as colorless viscous liquid.

$\mathrm{R}_{\mathrm{f}}: 0.50$ (hexane/ethyl acetate $=6: 4$ ).

IR (KBr) $v_{\max }: 3310,3067,2981,2935,1739,1708,1595$,

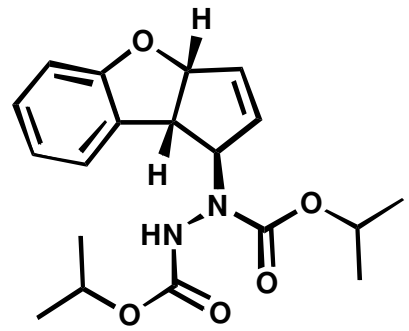
$1479,1311,1257,1146,1107,976,751 \mathrm{~cm}^{-1}$.

${ }^{1} \mathrm{H}$ NMR (300 MHz, $\left.\mathrm{CDCl}_{3}\right): \delta 7.31$ (brs, $\left.1 \mathrm{H}\right), 7.05(\mathrm{t}, 1 \mathrm{H}$, $J=7.40 \mathrm{~Hz}), 6.79(\mathrm{t}, 1 \mathrm{H}, J=7.30 \mathrm{~Hz}), 6.67-6.64(\mathrm{~d}, 1 \mathrm{H}, J$ $=7.91 \mathrm{~Hz}), 6.23(\mathrm{~s}, 1 \mathrm{H}), 6.00(\mathrm{~s}, 1 \mathrm{H}), 5.79-5.73(\mathrm{~m}, 2 \mathrm{H})$, $5.26(\mathrm{~s}, 1 \mathrm{H}), 4.96-4.87(\mathrm{~m}, 2 \mathrm{H}), 4.03(\mathrm{~s}, 1 \mathrm{H}), 1.25-1.19(\mathrm{~m}$, $12 \mathrm{H})$.

${ }^{13} \mathrm{C}$ NMR (125 MHz, $\left.\mathrm{CDCl}_{3}\right): \delta 157.9,156.1,155.1,132.6$, $128.8,128.2,125.3,120.7,110.0,90.8,71.3,70.4,69.9$, $50.1,22.1,21.9$. 
HRMS (EI): Calcd for $\mathrm{C}_{19} \mathrm{H}_{24} \mathrm{~N}_{2} \mathrm{O}_{5}, \mathrm{M}^{+}: 360.1685$; Found: 360.1695 .

\section{Di-tert-butyl 1-((1R,3aR,8bS)-3a,8b-dihydro-1H-benzo[b]cyclopenta[d]furan-1-} yl)hydrazine-1,2-dicarboxylate (3c).

Yield : $86 \%$ as white solid

$\mathrm{R}_{\mathrm{f}}: 0.52$ (hexane/ethyl acetate $=6: 4$ ).

Mp : $142-144{ }^{\circ} \mathrm{C}$

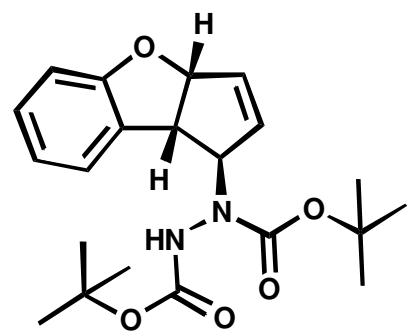

IR (KBr) $v_{\max }: 3396,2981,2935,1737,1703,1595,1477$, $1332,1240,1149,1107,966,856,751 \mathrm{~cm}^{-1}$.

${ }^{1} \mathrm{H}$ NMR $\left(300 \mathrm{MHz}, \mathrm{CDCl}_{3}\right): \delta 7.39$ (brs, $\left.1 \mathrm{H}\right), 7.11(\mathrm{t}, 1 \mathrm{H}$, $J=7.40 \mathrm{~Hz}), 6.86(\mathrm{t}, 1 \mathrm{H}, J=7.30 \mathrm{~Hz}), 6.74-6.71(\mathrm{~d}, 1 \mathrm{H}, J$ $=7.90 \mathrm{~Hz}), 6.07(\mathrm{~s}, 2 \mathrm{H}), 5.81(\mathrm{~m}, 2 \mathrm{H}), 5.31(\mathrm{~s}, 1 \mathrm{H}), 4.12$ (s, 1H), 1.52 (s, 9H), 1.47 (s, 9H).

${ }^{13} \mathrm{C}$ NMR $\left(125 \mathrm{MHz}, \mathrm{CDCl}_{3}\right): \delta 158.0,155.7,154.6,132.7$, $128.9,125.6,125.1,120.8,110.1,90.0,81.2,71.2,70.9$, $50.3,28.2$.

HRMS (EI): Calcd for $\mathrm{C}_{21} \mathrm{H}_{28} \mathrm{~N}_{2} \mathrm{O}_{5}, \mathrm{M}^{+}: 388.1998$; Found: 388.1995.

\section{Dibenzyl 1-((1R,3aR,8bS)-3a,8b-dihydro-1H-benzo[b]cyclopenta[d]furan-1-} yl)hydrazine-1,2-dicarboxylate (3d).

Yield : $87 \%$ as colorless viscous liquid.

$\mathrm{R}_{\mathrm{f}}: 0.48$ (hexane/ethyl acetate $=6: 4$ ).

IR (KBr) $v_{\max }: 3394,3031,2981,2927,1753,1714$,

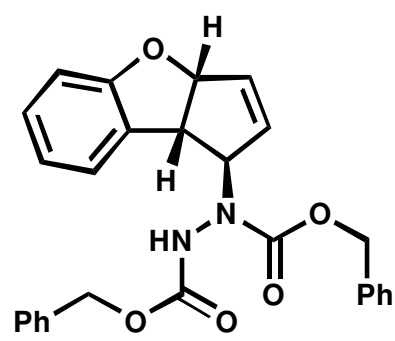
$1598,1460,1330,1255,1130,1103,968 \mathrm{~cm}^{-1}$.

${ }^{1} \mathrm{H}$ NMR $\left(300 \mathrm{MHz}, \mathrm{CDCl}_{3}\right): \delta 7.32(\mathrm{~m}, 11 \mathrm{H}), 7.09(\mathrm{t}$, $1 \mathrm{H}, J=7.30 \mathrm{~Hz}), 6.71-6.68(\mathrm{~d}, 2 \mathrm{H}, J=7.90 \mathrm{~Hz})$, 6.67 (brs, 1H), 6.03 (s, 1H), 5.82-5.73 (m, 2H), 5.34 (s, 1H), 5.20-5.12 (m, 4H), 4.09-4.05 (m, 1H). ${ }^{13} \mathrm{C}$ NMR $\left(125 \mathrm{MHz}, \mathrm{CDCl}_{3}\right): \delta 157.3,156.0,154.5$, $135.1,133.5,131.5,128.5,128.2,128.1,127.8,120.4$, 
114.3, 113.9, 109.6, 90.3, 71.4, 67.5, 55.0, 49.9 .

HRMS (EI): Calcd for $\mathrm{C}_{27} \mathrm{H}_{24} \mathrm{~N}_{2} \mathrm{O}_{5}, \mathrm{M}^{+}$: 456.1685;

Found: 456.1615.

\section{Diethyl 1-((1R,3aR,8bS)-7-phenyl-3a,8b-dihydro-1H-benzo[b]cyclopenta[d]furan-1- yl)hydrazine-1,2-dicarboxylate (3e)}

Yield : $78 \%$ as colorless viscous liquid.

$\mathrm{R}_{\mathrm{f}}: 0.40$ (hexane/ethyl acetate $=6: 4$ ).

IR (KBr) $v_{\max }: 3297,3058,3025,2972,2932,1750$,

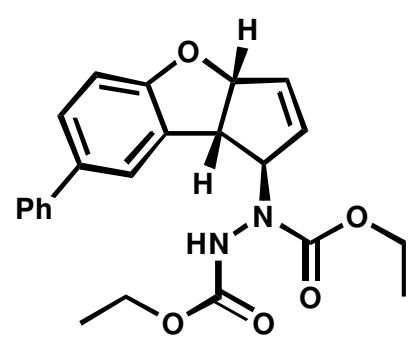
$1718,1606,1474,1314,1255,970,758 \mathrm{~cm}^{-1}$.

${ }^{1} \mathrm{H}$ NMR $\left(300 \mathrm{MHz}, \mathrm{CDCl}_{3}\right): \delta$ 7.61-7.28 (m, 7H), 6.84$6.81(\mathrm{~d}, 1 \mathrm{H}, J=8.18 \mathrm{~Hz}), 6.45(\mathrm{~s}, 1 \mathrm{H}), 6.14(\mathrm{~s}, 1 \mathrm{H})$, $5.91(\mathrm{~s}, 2 \mathrm{H}), 5.42(\mathrm{~s}, 1 \mathrm{H}), 4.29-4.20(\mathrm{~m}, 5 \mathrm{H}), 1.35-1.29$ $(\mathrm{m}, 6 \mathrm{H})$.

${ }^{13} \mathrm{C}$ NMR $\left(125 \mathrm{MHz}, \mathrm{CDCl}_{3}\right): \delta$ 157.7, 156.6, 155.6, $141.2,134.5,132.3,128.9,128.6,128.1,126.8,126.5$, $124.1,110.3,91.4,71.8,62.8,62.2,50.3,14.6,14.5$. HRMS (EI): Calcd for $\mathrm{C}_{23} \mathrm{H}_{24} \mathrm{~N}_{2} \mathrm{O}_{5}, \mathrm{M}^{+}$: 408.1685; Found: 408.1693.

Yield : $81 \%$ as colorless viscous liquid.

$\mathrm{R}_{\mathrm{f}}: 0.46$ (hexane/ethyl acetate $=6: 4$ ).

IR (KBr) $v_{\max }: 3303,3065,3025,2979,2919,1706$,

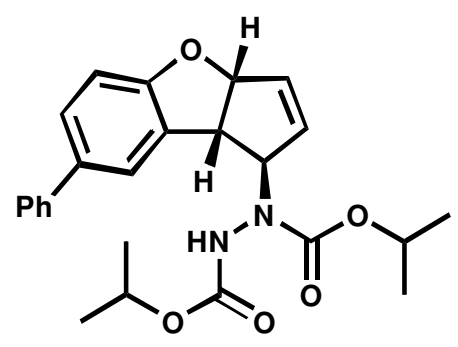
$1600,1474,1374,1248,1102,956,758 \mathrm{~cm}^{-1}$.

${ }^{1} \mathrm{H}$ NMR (300 MHz, $\left.\mathrm{CDCl}_{3}\right): \delta$ 7.60-7.25 (m, 7H), 6.81$6.78(\mathrm{~d}, 1 \mathrm{H}, J=8.29 \mathrm{~Hz}), 6.26(\mathrm{~s}, 1 \mathrm{H}), 6.11(\mathrm{~s}, 1 \mathrm{H}), 5.88$ (s, 2H), $5.39(\mathrm{~s}, 1 \mathrm{H}), 5.04-4.93(\mathrm{~m}, 2 \mathrm{H}), 4.17(\mathrm{~s}, 1 \mathrm{H})$, 1.32-1.27 (m, 12H).

${ }^{13} \mathrm{C}$ NMR (125 MHz, $\left.\mathrm{CDCl}_{3}\right): \delta 157.7,156.2,155.1$, $141.2,134.5,132.5,128.9,128.6,128.0,126.8,126.4$, 124.1, 110.2, 91.4, 70.3, 69.9, 50.2, 22.1, 21.9 . 
HRMS (EI): Calcd for $\mathrm{C}_{25} \mathrm{H}_{28} \mathrm{~N}_{2} \mathrm{O}_{5}, \mathrm{M}^{+}$: 436.1998;

Found: 436.2030 .

\section{Di-tert-butyl}

1-((1R,3aR,8bS)-7-phenyl-3a,8b-dihydro-1H-

\section{benzo[b]cyclopenta[d]furan-1-yl)hydrazine-1,2-dicarboxylate (3g).}

Yield : $80 \%$ as colorless viscous liquid.

$\mathrm{R}_{\mathrm{f}}: 0.44$ (hexane/ethyl acetate $=6: 4$ ).

IR (KBr) $v_{\max }: 3330,3051,2976,2927,1745,1706$,

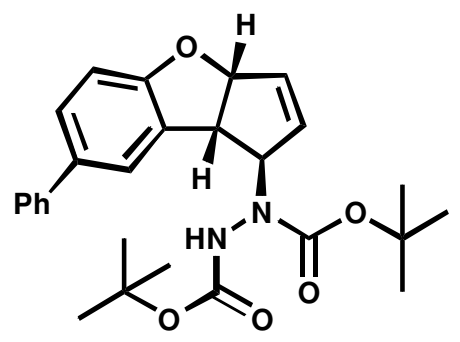

$1613,1470,1327,1253,1160,966,751 \mathrm{~cm}^{-1}$.

${ }^{1} \mathrm{H}$ NMR $\left(300 \mathrm{MHz}, \mathrm{CDCl}_{3}\right): \delta$ 7.59-7.28 (m, 7H), 6.81-6.78 (d, 1H, $J=8.22 \mathrm{~Hz}), 6.09(\mathrm{~s}, 2 \mathrm{H}), 5.87(\mathrm{~s}$, 2H), $5.36(\mathrm{~s}, 1 \mathrm{H}), 4.16(\mathrm{~s}, 1 \mathrm{H}), 1.52(\mathrm{~s}, 9 \mathrm{H}), 1.48(\mathrm{~s}$, 9H).

${ }^{13} \mathrm{C}$ NMR $\left(125 \mathrm{MHz}, \mathrm{CDCl}_{3}\right): \delta$ 157.6, 155.6, 154.4, $141.2,134.4,129.1,128.5,127.9,126.8,126.4,124.1$, $110.1,91.4,81.2,50.0,28.3,28.1$.

HRMS (EI): Calcd for $\mathrm{C}_{27} \mathrm{H}_{32} \mathrm{~N}_{2} \mathrm{O}_{5}, \mathrm{M}^{+}$: 464.2311; Found: 464.2310.

\section{Dibenzyl 1-((1R,3aR,8bS)-7-phenyl-3a,8b-dihydro-1H-benzo[b]cyclopenta[d]furan-} 1-yl)hydrazine-1,2-dicarboxylate (3h).

Yield : $75 \%$ as colorless viscous liquid.

$\mathrm{R}_{\mathrm{f}}: 0.42$ (hexane/ethyl acetate $=6: 4$ ).

IR (KBr) $v_{\max }: 3297,3065,3032,2952,2919,1719$,

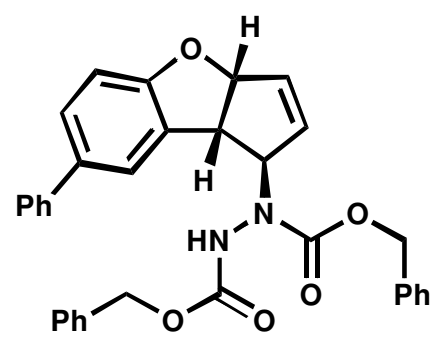
$1600,1474,1248,1314,1129,970,751 \mathrm{~cm}^{-1}$.

${ }^{1} \mathrm{H}$ NMR (300 MHz, $\left.\mathrm{CDCl}_{3}\right): \delta$ 7.70-7.31 (m, 17H), 6.78-6.76 (d, 1H, J = 8.31 Hz), 6.65 (brs, 1H), 6.04 (s, 1H), 5.84-5.79 (m, 2H), 5.42 (s, 1H), 5.26-5.12 (m, 4H), 4.21-4.07 (m, 1H).

${ }^{13} \mathrm{C}$ NMR (125 MHz, $\left.\mathrm{CDCl}_{3}\right): \delta$ 157.6, 156.4, 155.4, 141.2 , 135.5, 134.5, 132.2, 128.6, 128.5, 128.2, 126.9, 124.1, 110.3, 91.3, 71.7, 67.9, 50.2. 
MS (FAB): Calcd for $\mathrm{C}_{33} \mathrm{H}_{28} \mathrm{~N}_{2} \mathrm{O}_{5},(\mathrm{M}+1)$ : 533.1998;

Found: 533.2007.

Diethyl 1-((1R,3aR,8bS)-7-acetyl-3a,8b-dihydro-1H-benzo[b]cyclopenta[d]furan-1yl)hydrazine-1,2-dicarboxylate (3i).

Yield : $60 \%$ as colorless viscous liquid.

$\mathrm{R}_{\mathrm{f}}: 0.15$ (hexane/ethyl acetate $=6: 4$ ).

IR (KBr) $v_{\max }: 3299,2987,2912,2839,1734,1706$,

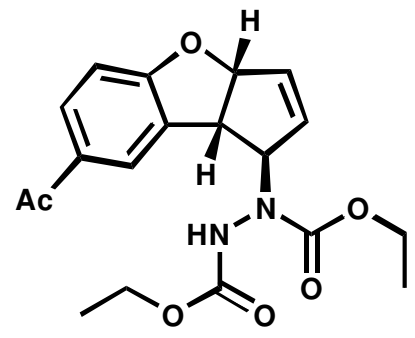
$1679,1604,1487,1261,1116,950,764 \mathrm{~cm}^{-1}$.

${ }^{1} \mathrm{H}$ NMR $\left(300 \mathrm{MHz}, \mathrm{CDCl}_{3}\right): \delta 8.06(\mathrm{~s}, 1 \mathrm{H}), 7.86-7.83$

$(\mathrm{d}, 1 \mathrm{H}, J=8.40 \mathrm{~Hz}), 6.80-6.77(\mathrm{~d}, 1 \mathrm{H}, J=8.40 \mathrm{~Hz})$, $6.36(\mathrm{~s}, 1 \mathrm{H}), 6.13(\mathrm{~m}, 1 \mathrm{H}), 5.97-5.94(\mathrm{~m}, 2 \mathrm{H}), 5.35(\mathrm{~s}$, $1 \mathrm{H}), 4.33-4.19(\mathrm{~m}, 5 \mathrm{H}), 2.58(\mathrm{~s}, 3 \mathrm{H}), 1.39-1.27(\mathrm{~m}$, $6 \mathrm{H})$.

${ }^{13} \mathrm{C}$ NMR $\left(125 \mathrm{MHz}, \mathrm{CDCl}_{3}\right): \delta 196.0,162.3,156.8$, 155.6, 134.8, 131.1, 129.0, 126.1, ,109.8, 92.4, 71.9, 62.3, 49.7, 14.6, 14.4 .

MS (FAB): Calcd for $\mathrm{C}_{19} \mathrm{H}_{22} \mathrm{~N}_{2} \mathrm{O}_{6},(\mathrm{M}+1)$ : 375.1478;

Found: 375.1500 .

\section{Diisopropyl}

1-((1R,3aR,8bS)-7-acetyl-3a,8b-dihydro-1Hbenzo[b]cyclopenta[d]furan-1-yl)hydrazine-1,2-dicarboxylate (3j).

Yield : 55\% as colorless viscous liquid.

$\mathrm{R}_{\mathrm{f}}: 0.19$ (hexane/ethyl acetate $=6: 4$ ).

IR (KBr) $v_{\max }: 3297,3058,2979,2925,1743,1712$,

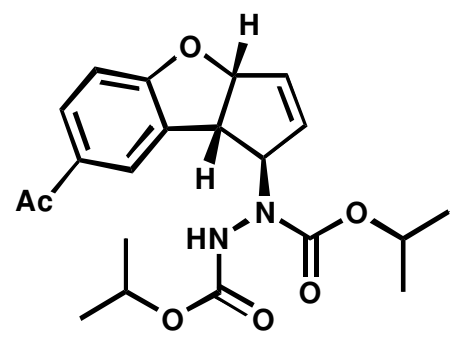
$1650,1601,1487,1261,1109,970,771 \mathrm{~cm}^{-1}$.

${ }^{1} \mathrm{H}$ NMR $\left(300 \mathrm{MHz}, \mathrm{CDCl}_{3}\right): \delta 7.99(\mathrm{~s}, 1 \mathrm{H}), 7.79-7.77(\mathrm{~d}$, $1 \mathrm{H}, J=8.50 \mathrm{~Hz}), 6.66(\mathrm{~m}, 1 \mathrm{H}), 6.22(\mathrm{~s}, 1 \mathrm{H}), 6.03(\mathrm{~s}, 1 \mathrm{H})$, 5.84-5.81 (m, 2H), $5.23(\mathrm{~s}, 1 \mathrm{H}), 4.95-4.86(\mathrm{~m}, 2 \mathrm{H}), 4.09$ (s, 1H), 2.49 (s, 3H), 1.21-1.20 (m, 12H).

${ }^{13} \mathrm{C}$ NMR $\left(125 \mathrm{MHz}, \mathrm{CDCl}_{3}\right): \delta 199.4,161.9,156.2$, 154.9, 143.9, 133.8, 130.8, 127.9, 120.8, 109.6, 92.6, 72.1, 69.7, 49.9, 22.1, 21.9. 
MS (FAB): Calcd for $\mathrm{C}_{21} \mathrm{H}_{26} \mathrm{~N}_{2} \mathrm{O}_{6},(\mathrm{M}+1)$ : 403.1791;

Found: 403.1752.

\section{Diethyl}

dicarboxylate (4).

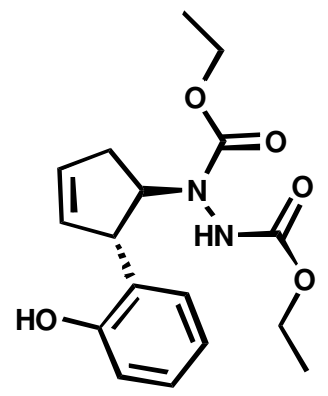

1-((1S,2S)-2-(2-hydroxyphenyl)cyclopent-3-enyl)hydrazine-1,2-

Yield : $80 \%$ as colorless viscous liquid.

$\mathrm{R}_{\mathrm{f}}: 0.40$ (hexane/ethyl acetate $=6: 4$ ).

IR (KBr) $v_{\max }: 3443,3290,3051,2959,2919,2846$, 1744, 1719, 1600, 1454, 1235, 1155, 1049, 950, 751 $\mathrm{cm}^{-1}$.

${ }^{1} \mathrm{H}$ NMR (300 MHz, $\mathrm{CDCl}_{3}$ ): $\delta$ 7.31-7.17 (m, 5H), 6.37 $(\mathrm{s}, 1 \mathrm{H}), 5.87-5.85(\mathrm{~m}, 1 \mathrm{H}), 5.72-5.69(\mathrm{~m}, 1 \mathrm{H}), 4.75-$ $4.72(\mathrm{~m}, 1 \mathrm{H}), 4.26-4.19$ (m, 2H), 4.00 (s, 3H), 2.68-2.58 (m, 2H), 1.33-1.25 (m, 3H), 1.06 (s, 3H). ${ }^{13} \mathrm{C}$ NMR $\left(125 \mathrm{MHz}, \mathrm{CDCl}_{3}\right): \delta$ 156.7, 155.9, 143.2, $132.7,129.6,128.4,127.4,126.5,67.2$, 62.2, 62.1, 53.8, $35.1,14.4,14.2$.

HRMS (FAB): Calcd for $\mathrm{C}_{17} \mathrm{H}_{22} \mathrm{~N}_{2} \mathrm{O}_{5}, \quad(\mathrm{M}+1)$ : 335.1529; Found: 335.1502.

\section{Diethyl 1-((1S,2S)-2-(2-aminophenyl)cyclopent-3-enyl)hydrazine-1,2-dicarboxylate} (6).

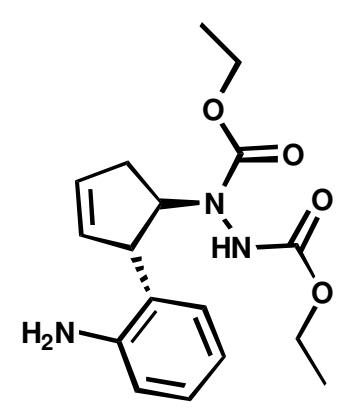

Yield : $60 \%$ as colorless viscous liquid.

$\mathrm{R}_{\mathrm{f}}: 0.23$ (hexane/ethyl acetate $=6: 4$ ).

IR (KBr) $v_{\max }: 3363,2959,2919,2846,1738,1714$, 1604, 1462, 1259, 1023, 869, $798 \mathrm{~cm}^{-1}$.

${ }^{1} \mathrm{H}$ NMR $\left(500 \mathrm{MHz}, \mathrm{CDCl}_{3}\right): \delta 7.59-7.58(\mathrm{~m}, 1 \mathrm{H}), 7.41-$ $7.39(\mathrm{~m}, 1 \mathrm{H}), 6.94-6.86(\mathrm{~m}, 2 \mathrm{H})$ 6.59-6.56 (m, 3H), $5.84(\mathrm{~s}, 1 \mathrm{H}), 5.66(\mathrm{~s}, 1 \mathrm{H}), 4.68-4.66(\mathrm{~m}, 1 \mathrm{H}), 4.16-4.13$ (m, 2H), 4.05-4.02 (m, 3H), 2.67 (m, 1H), 2.42-2.40 (m, $1 \mathrm{H}),(1.24-1.22$ (m, 3H), 1.08-1.02 (m, 3H). 
${ }^{13} \mathrm{C}$ NMR $\left(125 \mathrm{MHz}, \mathrm{CDCl}_{3}\right): \delta 155.6,154.1,132.7$, $132.1,131.9,128.5,128.4,127.4,62.5,62.3,49.4,36.1$, 14.5.

HRMS (EI): Calcd for $\mathrm{C}_{17} \mathrm{H}_{23} \mathrm{~N}_{3} \mathrm{O}_{4}, \mathrm{M}^{+}$: 333.1689;

Found: 333.1680 .

\section{Diethyl 1-((1R,3aR,8bR)-1,3a,4,8b-tetrahydrocyclopenta[b]indol-1-yl)hydrazine-1,2- dicarboxylate (7a).}

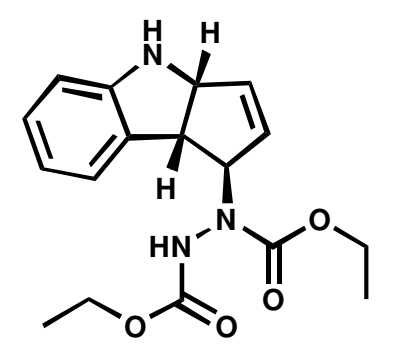

Yield : 93\% as colorless viscous liquid.

$\mathrm{R}_{\mathrm{f}}: 0.15$ (hexane/ethyl acetate $=6: 4$ ).

IR (KBr) $v_{\max }: 3297,2979,2919,2859,1712,1606$, $1414,1228,1056,943,758 \mathrm{~cm}^{-1}$.

${ }^{1} \mathrm{H}$ NMR (300 MHz, $\left.\mathrm{CDCl}_{3}\right): \delta 7.26$ (brs, $\left.1 \mathrm{H}\right), 7.03(\mathrm{t}$, $1 \mathrm{H}, J=7.50 \mathrm{~Hz}), 6.75(\mathrm{t}, 1 \mathrm{H}, J=7.40 \mathrm{~Hz}), 6.60-6.58$ $(\mathrm{d}, 1 \mathrm{H}, J=7.90 \mathrm{~Hz}), 6.49$ (s, 1H), 6.39 (s, 1H), 5.88 (m, $1 \mathrm{H}), 5.75(\mathrm{~m}, 1 \mathrm{H}), 5.35(\mathrm{~s}, 1 \mathrm{H}), 4.90-4.88(\mathrm{~m}, 1 \mathrm{H})$, 4.26-4.10 (m, 4H), 4.01 (m, 1H), 1.34-1.26 (m, 6H).

${ }^{13} \mathrm{C}$ NMR $\left(125 \mathrm{MHz}, \mathrm{CDCl}_{3}\right): \delta 155.6,154.6,148.5$, 139.2 , 130.6, 129.3, 128.3, 125.5, ,119.7, 110.7, 70.7, 67.6, 51.1, 14.2.

HRMS (EI): Calcd for $\mathrm{C}_{17} \mathrm{H}_{21} \mathrm{~N}_{3} \mathrm{O}_{4}, \mathrm{M}^{+}$: 331.1532; Found: 331.1500.

\section{Diisopropyl} yl)hydrazine-1,2-dicarboxylate (7b).

Yield : $85 \%$ as colorless viscous liquid.

$\mathrm{R}_{\mathrm{f}}: 0.17$ (hexane/ethyl acetate $=6: 4$ ).

IR (KBr) $v_{\max }: 3297,3051,2972,2925,1741,1705$, 1600, 1487, 1374, 1255, 1102, 956, $758 \mathrm{~cm}^{-1}$.

${ }^{1} \mathrm{H}$ NMR $\left(300 \mathrm{MHz}, \mathrm{CDCl}_{3}\right): \delta 7.26(\mathrm{~s}, 1 \mathrm{H}), 7.03(\mathrm{t}$, $1 \mathrm{H}, J=7.30 \mathrm{~Hz}), 6.76(\mathrm{t}, 1 \mathrm{H}, J=7.30 \mathrm{~Hz}), 6.61-6.58$ 


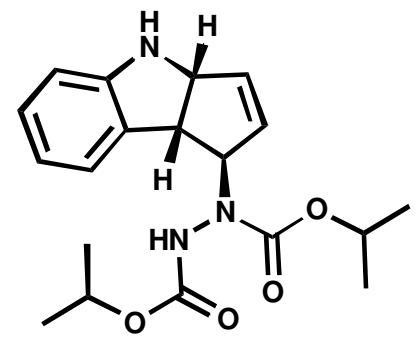

(d, $1 \mathrm{H}, J=7.80 \mathrm{~Hz}), 6.18(\mathrm{~s}, 1 \mathrm{H}), 5.88(\mathrm{~s}, 1 \mathrm{H}), 5.75(\mathrm{~s}$, $1 \mathrm{H}), 5.34(\mathrm{~m}, 1 \mathrm{H}), 5.03-4.91(\mathrm{~m}, 3 \mathrm{H}), 4.02(\mathrm{~m}, 1 \mathrm{H})$, $1.28-1.26(\mathrm{~d}, 12 \mathrm{H}, J=6.20 \mathrm{~Hz})$.

${ }^{13} \mathrm{C}$ NMR $\left(125 \mathrm{MHz}, \mathrm{CDCl}_{3}\right): \delta 156.3,155.3,148.5$, 130.3, 128.4, 125.6, 119.9, 110.9, 70.4, 69.8, 67.5, 51.3, 22.3, 22.0.

HRMS (EI): Calcd for $\mathrm{C}_{19} \mathrm{H}_{25} \mathrm{~N}_{3} \mathrm{O}_{4}, \mathrm{M}^{+}$: 359.1845; Found: 359.1838 .

\section{Di-tert-butyl} yl)hydrazine-1,2-dicarboxylate (7c).

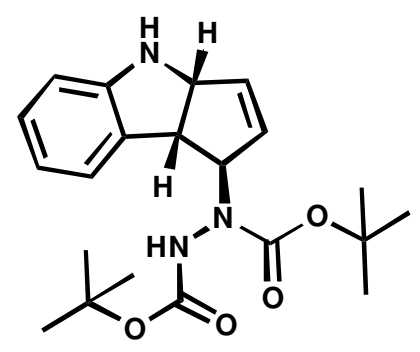

Yield : 75\% as white solid

$\mathrm{R}_{\mathrm{f}}: 0.19$ (hexane/ethyl acetate $=6: 4$ ).

IR (KBr) $v_{\max }: 3310,2959,2912,2851,1732,1704$, 1606, 1467, 1248, 1155, 950, $758 \mathrm{~cm}^{-1}$.

${ }^{1} \mathrm{H}$ NMR $\left(300 \mathrm{MHz}, \mathrm{CDCl}_{3}\right): \delta 7.26(\mathrm{~m}, 1 \mathrm{H}), 7.02(\mathrm{t}$, $1 \mathrm{H}, J=7.50 \mathrm{~Hz}), 6.75(\mathrm{t}, 1 \mathrm{H}, J=7.30 \mathrm{~Hz}), 6.59-6.57$ (d, 1H, $J=7.60 \mathrm{~Hz}), 6.08$ (s, 1H), $5.85(\mathrm{~m}, 1 \mathrm{H}), 5.76$ (m, 1H), $5.32(\mathrm{~s}, 1 \mathrm{H}), 4.89(\mathrm{~m}, 1 \mathrm{H}), 4.09-4.04(\mathrm{~m}, 1 \mathrm{H})$, $1.52(\mathrm{~s}, 9 \mathrm{H}), 1.47$ (s, 9H).

${ }^{13} \mathrm{C}$ NMR (125 MHz, $\left.\mathrm{CDCl}_{3}\right): \delta 155.2,154.2,148.2$, 134.3, 129.9, 127.7, 119.4, 110.2, 81.3, 80.4, 66.9, 52.7, 29.2, 27.7 .

MS (FAB): Calcd for $\mathrm{C}_{21} \mathrm{H}_{29} \mathrm{~N}_{3} \mathrm{O}_{4}$, (M+1): 388.2158;

Found: 388.2190. 
ORTEP drawing: 3c

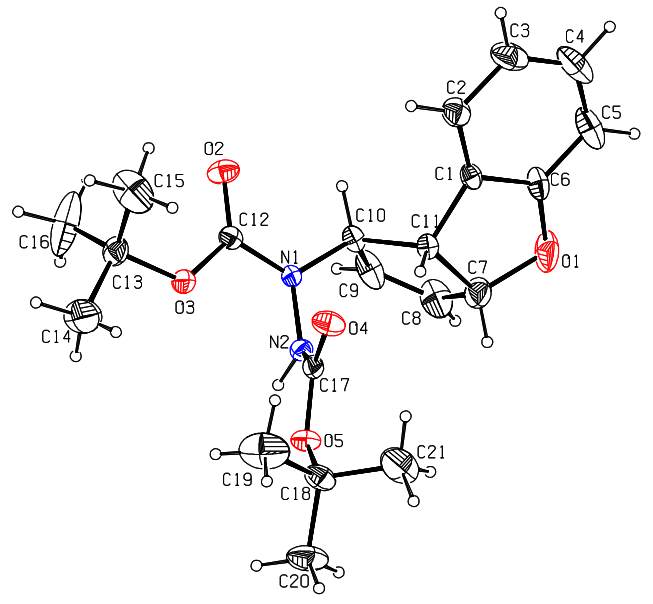

Empirical formula

$\mathrm{C}_{21} \mathrm{H}_{28} \mathrm{~N}_{2} \mathrm{O}_{5}$

Formula weight

388.45

Temperature

293(2) K

Wavelength

$0.71073 \mathrm{~A}$

Crystal system

Monoclinic

Space group

$\mathrm{P} 21 / \mathrm{c}$

Unit cell dimensions

$$
\begin{aligned}
& \mathrm{a}=11.853(4) \text { A } \text { alpha }=90 \mathrm{deg} . \\
& \mathrm{b}=19.502(7) \text { A } \text { beta }=106.351(7) \mathrm{deg} . \\
& \mathrm{c}=9.979(3) \text { A gamma }=90 \mathrm{deg} .
\end{aligned}
$$

Volume

$2213.5(13) \mathrm{A}^{\wedge} 3$

Z

4

Density (calculated)

$1.166 \mathrm{Mg} / \mathrm{m}^{\wedge} 3$

Absorption coefficient

$0.083 \mathrm{~mm}^{\wedge}-1$

$\mathrm{F}(000)$

832

Crystal size

$0.46 \times 0.31 \times 0.05 \mathrm{~mm}$

Theta range for data collection 1.79 to $25.00 \mathrm{deg}$.

Index ranges

$-11<=\mathrm{h}<=14,-23<=\mathrm{k}<=21,-11<=\mathrm{l}<=10$ 
Reflections collected $\quad 10991$

Independent reflections $\quad 3888[\mathrm{R}(\mathrm{int})=0.0623]$

Absorption correction Semi-empirical from equivalents

Max. and min. transmission $\quad 0.9958$ and 0.9627

Refinement method $\quad$ Full-matrix least-squares on $\mathrm{F}^{\wedge} 2$

Data / restraints / parameters 3888 / 0 / 263

Goodness-of-fit on $\mathrm{F}^{\wedge} 2$

Final $\mathrm{R}$ indices $[\mathrm{I}>2 \operatorname{sigma}(\mathrm{I})] \quad \mathrm{R} 1=0.1168, \mathrm{wR} 2=0.2187$

$\mathrm{R}$ indices (all data) $\quad \mathrm{R} 1=0.1741, \mathrm{wR} 2=0.2431$

Largest diff. peak and hole $\quad 0.217$ and -0.179 e. $\mathrm{A}^{\wedge}$-3 

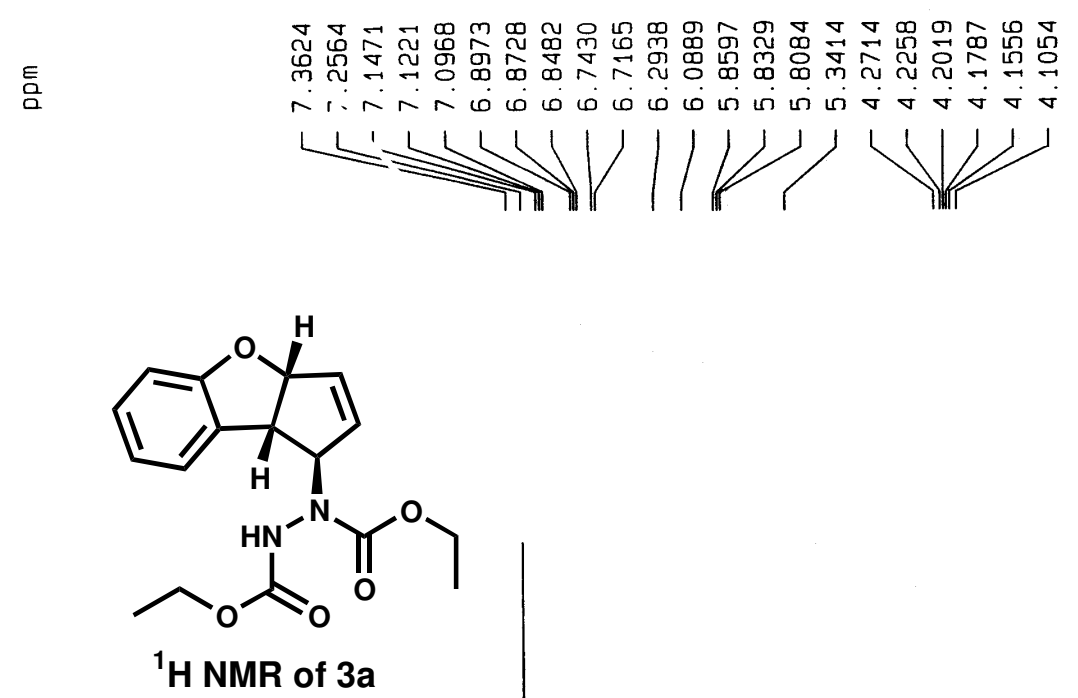

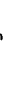

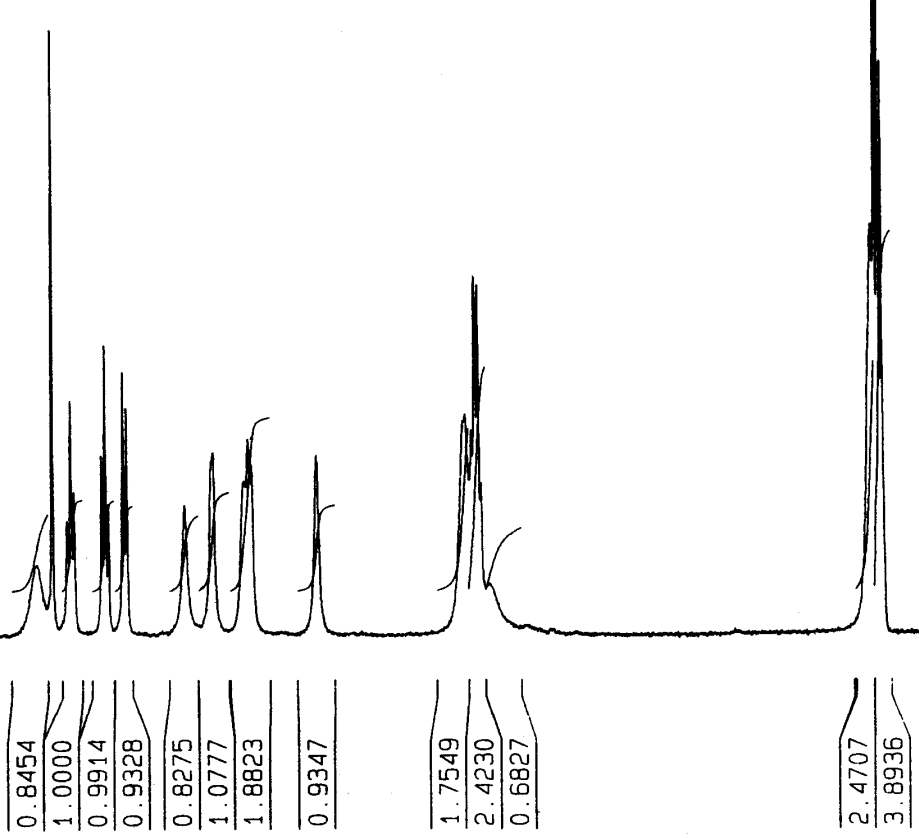




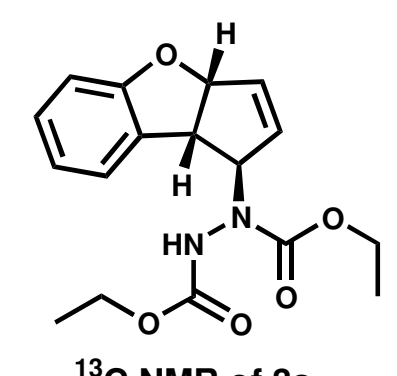

${ }^{13} \mathrm{C}$ NMR of $3 a$

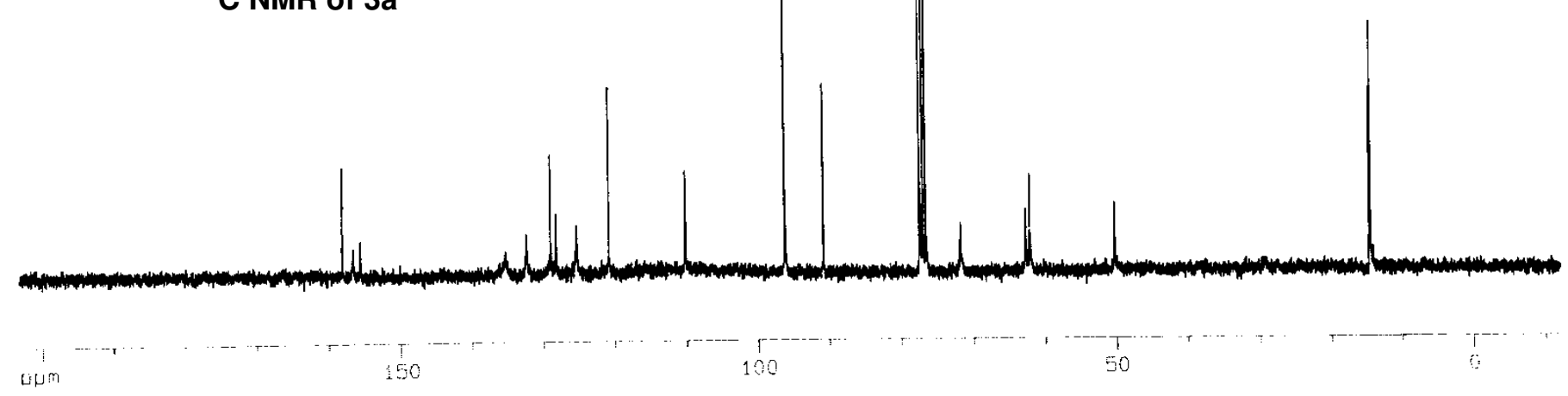



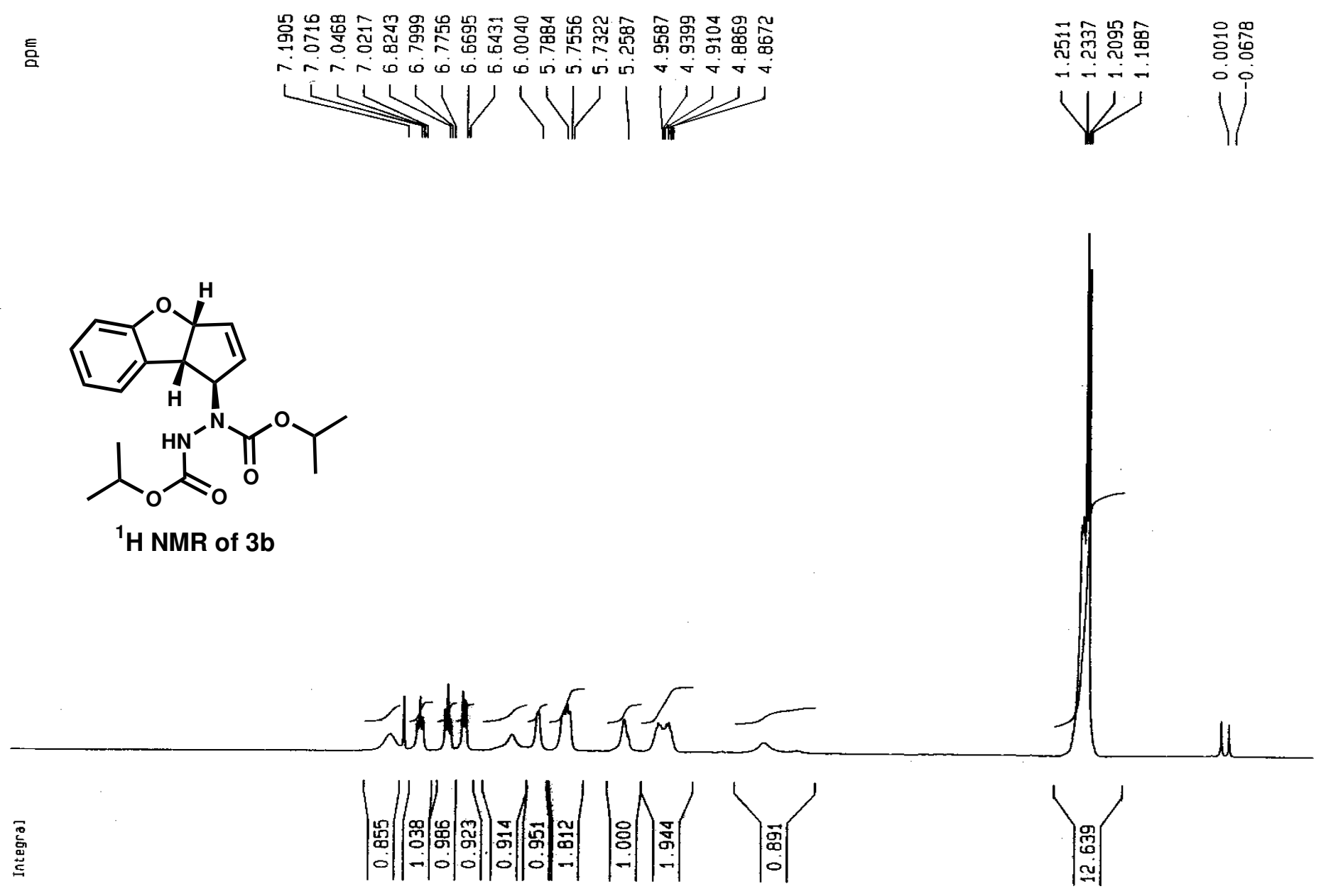

ppm 10
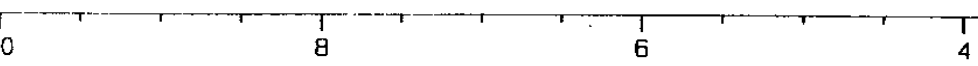


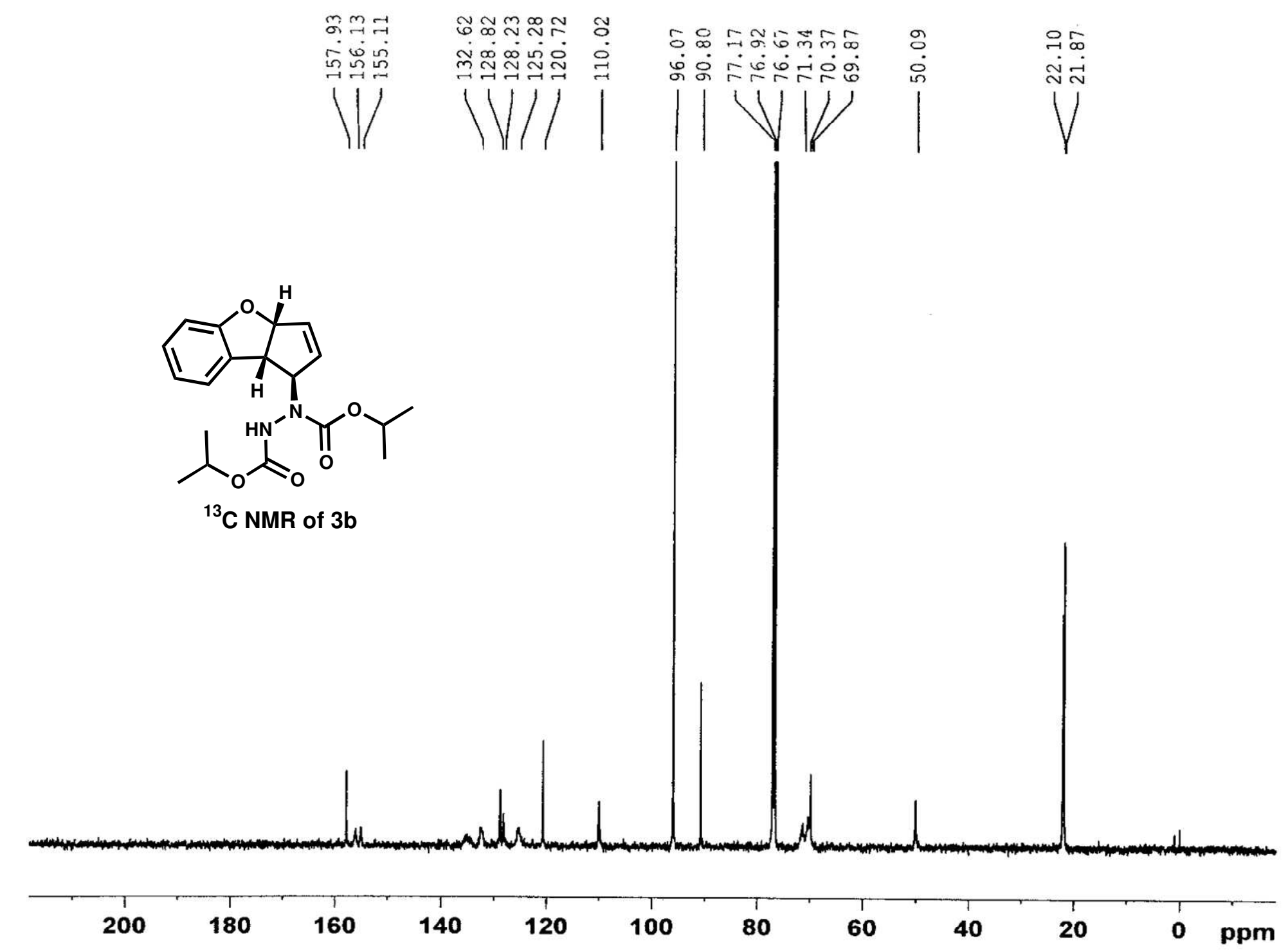




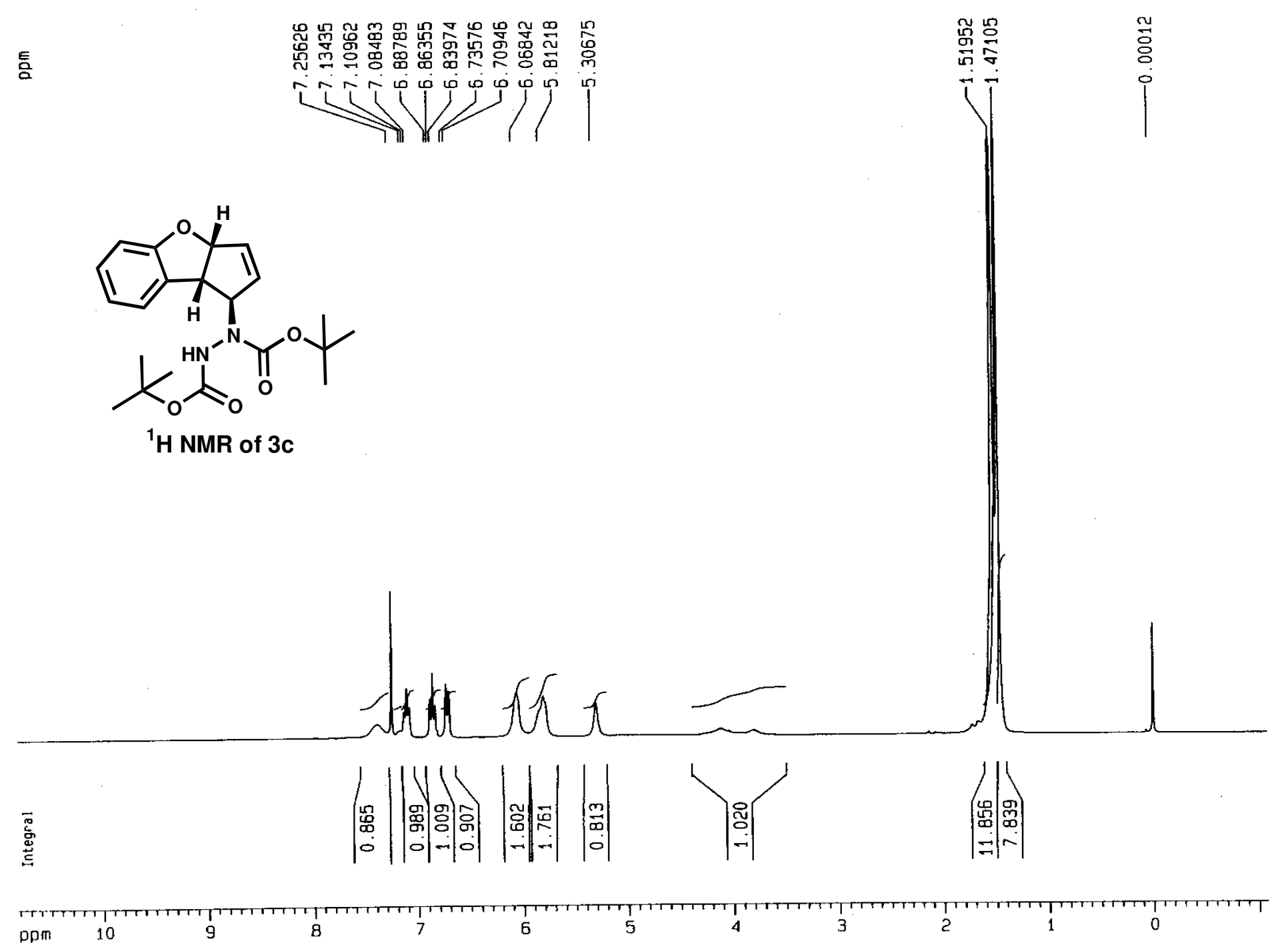

S21 


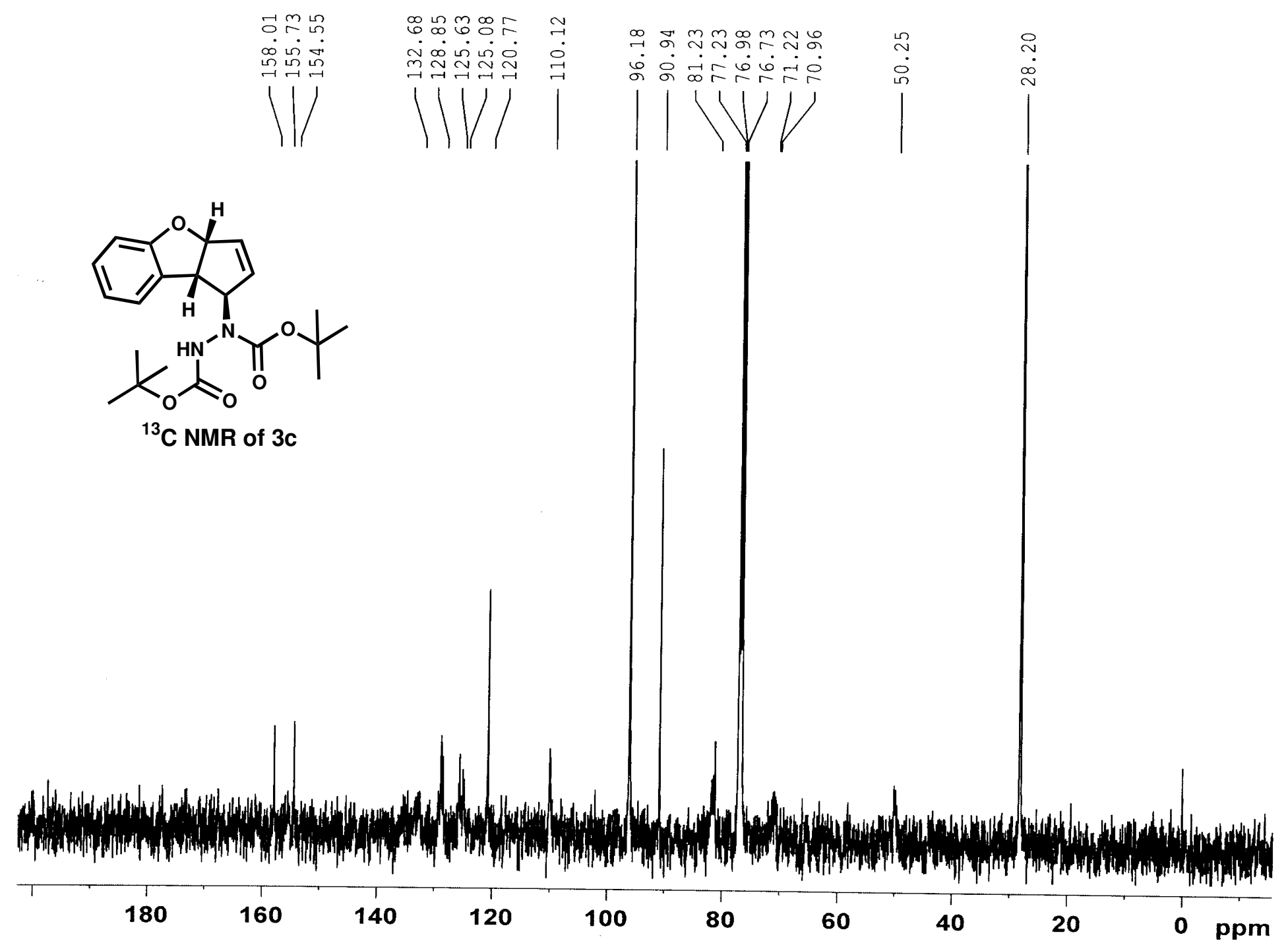




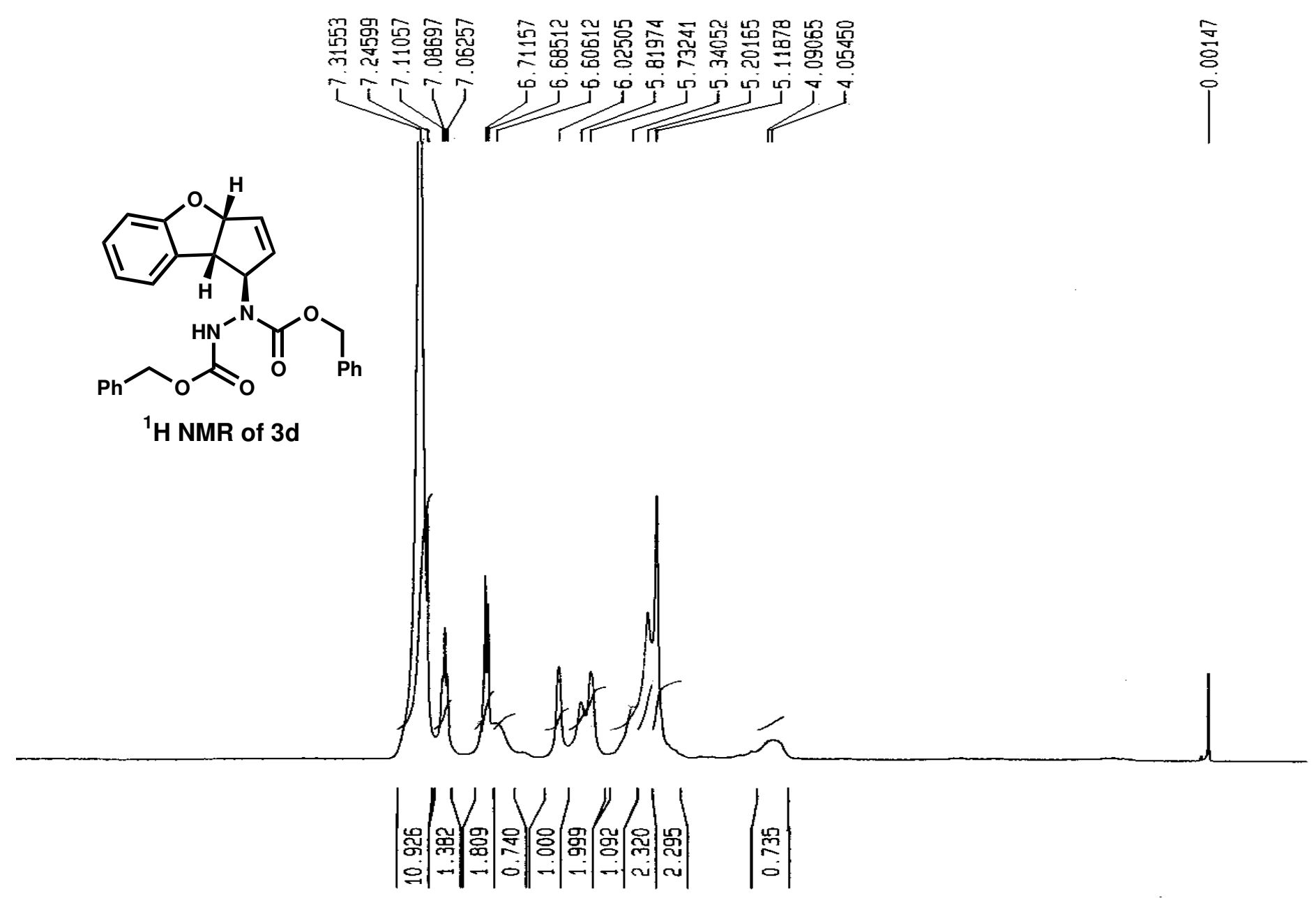




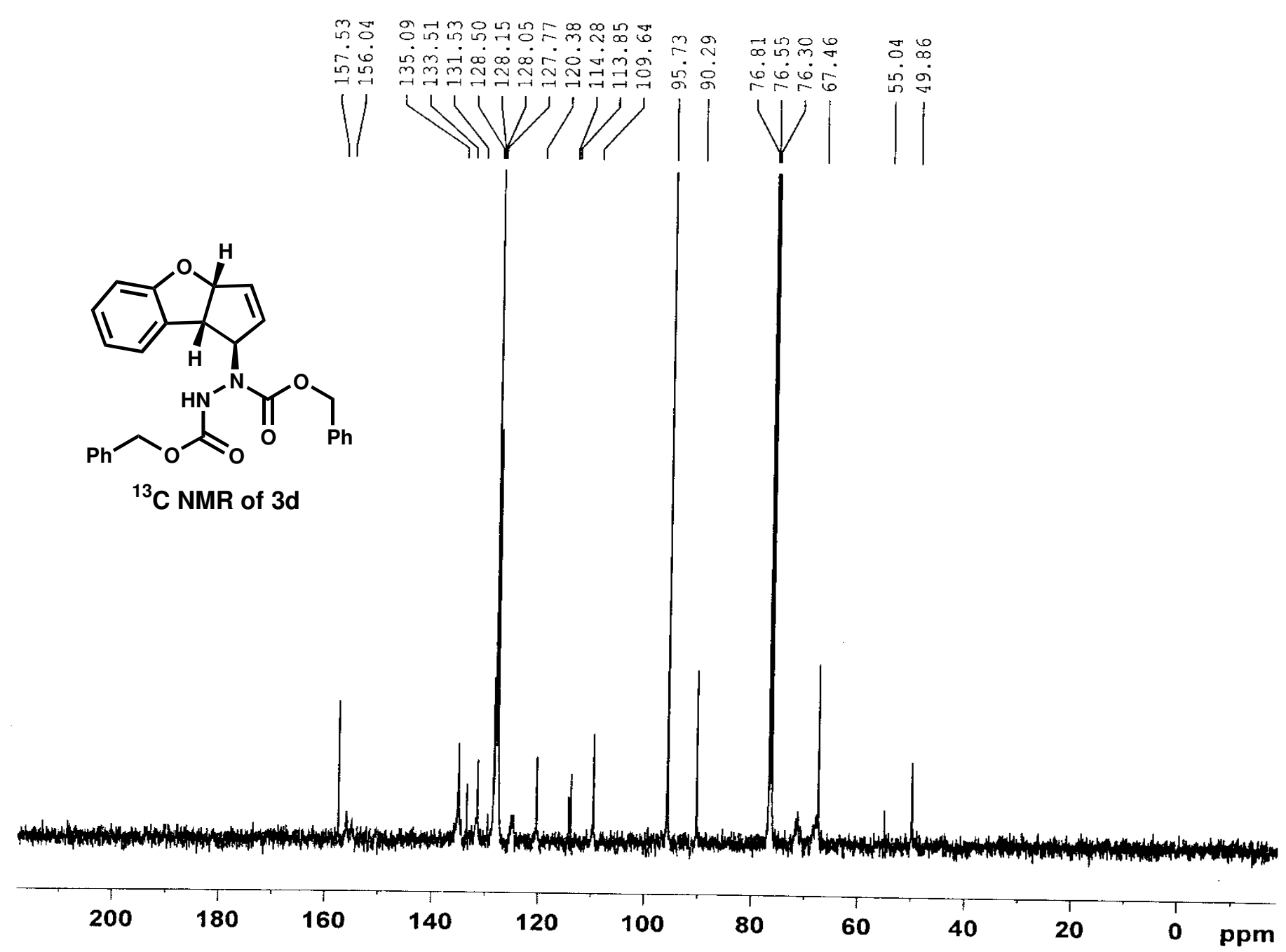




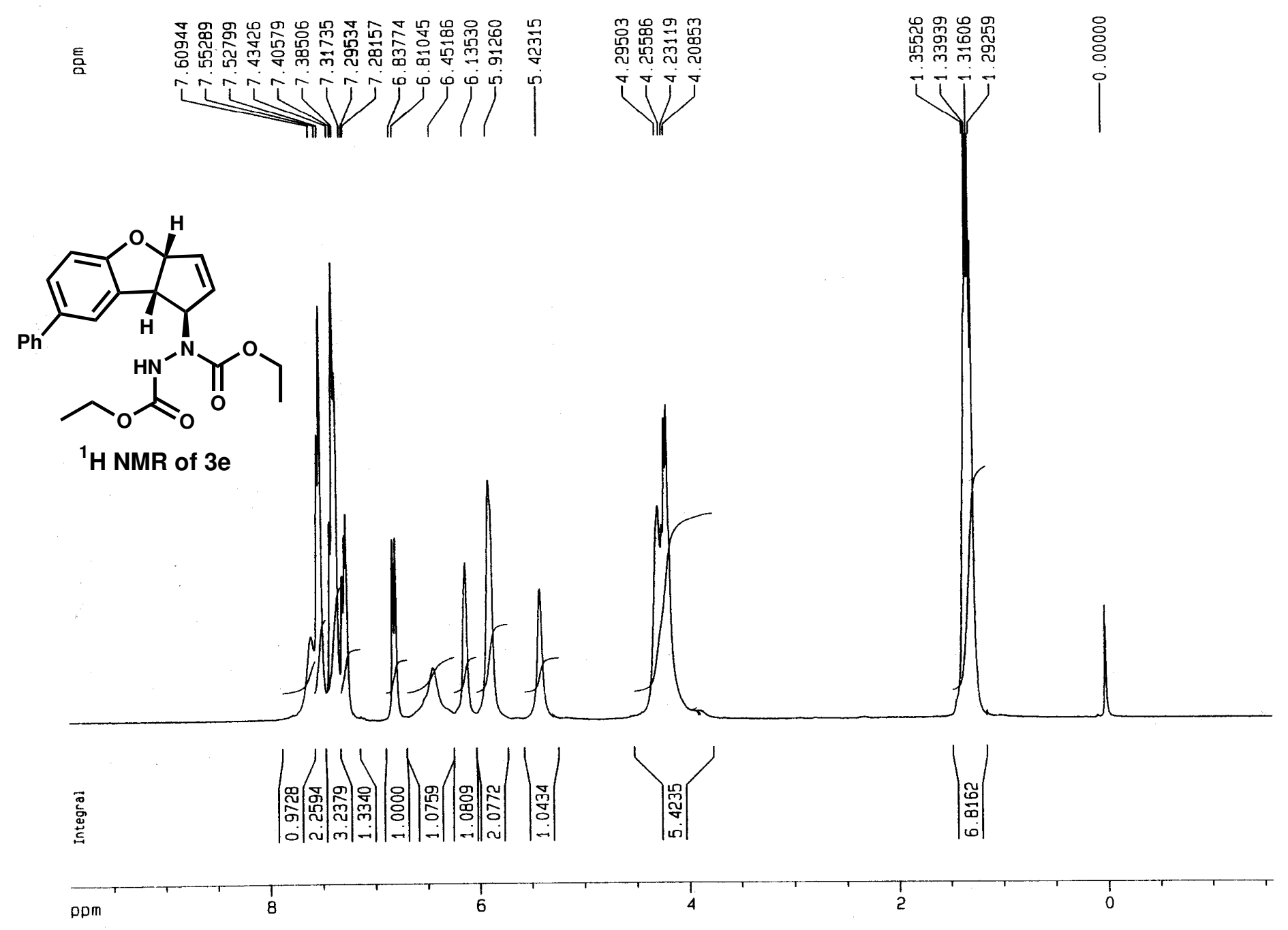




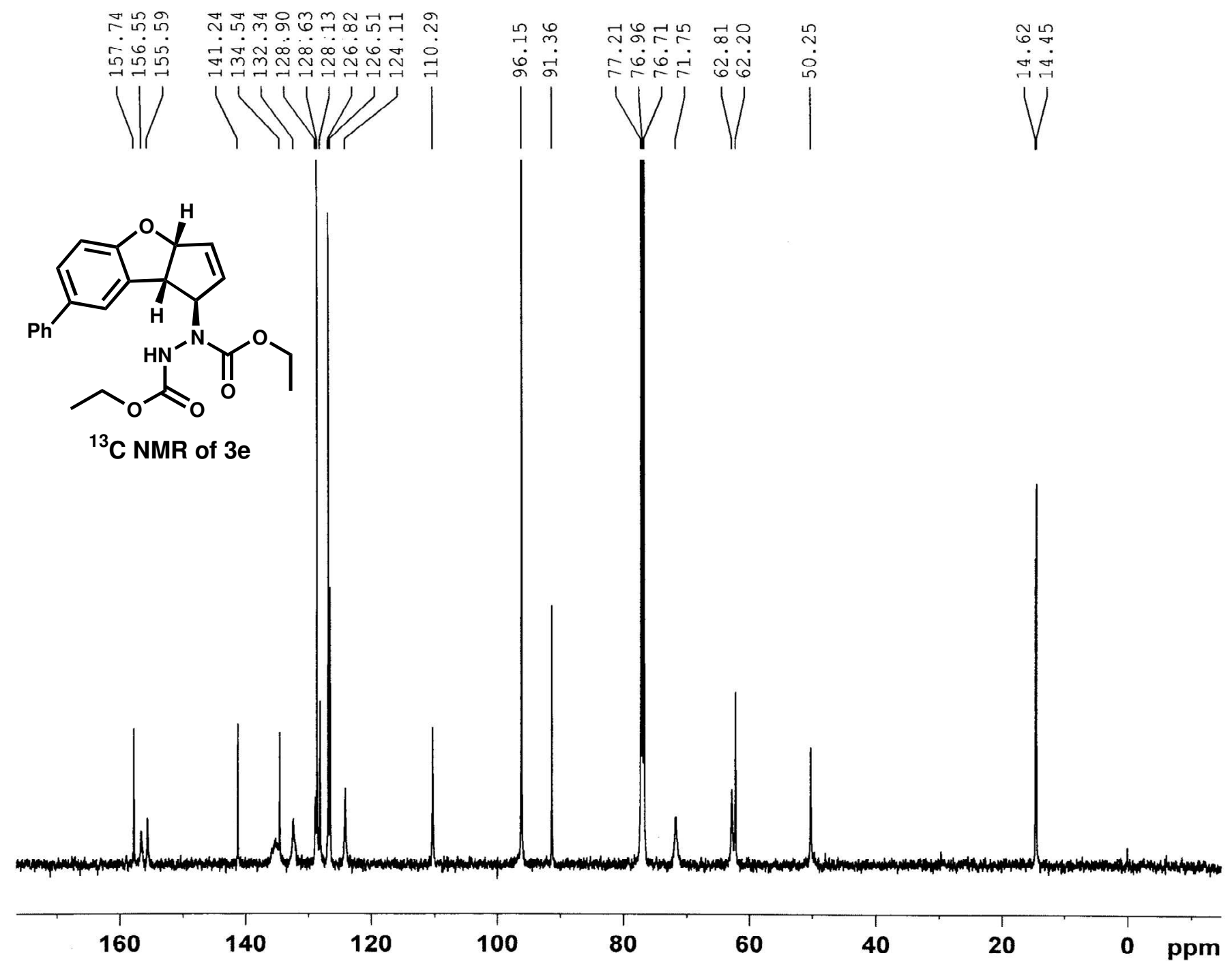




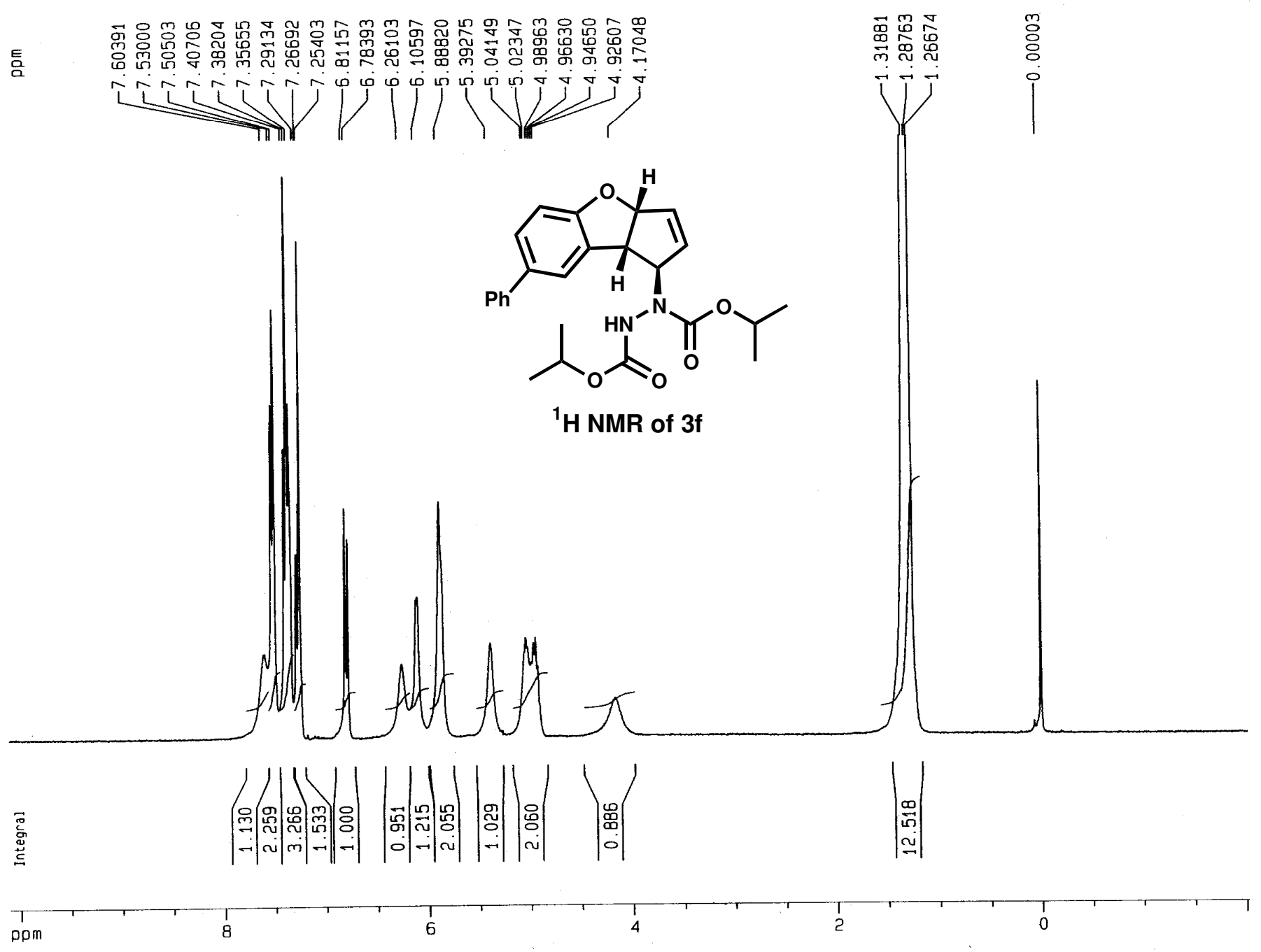




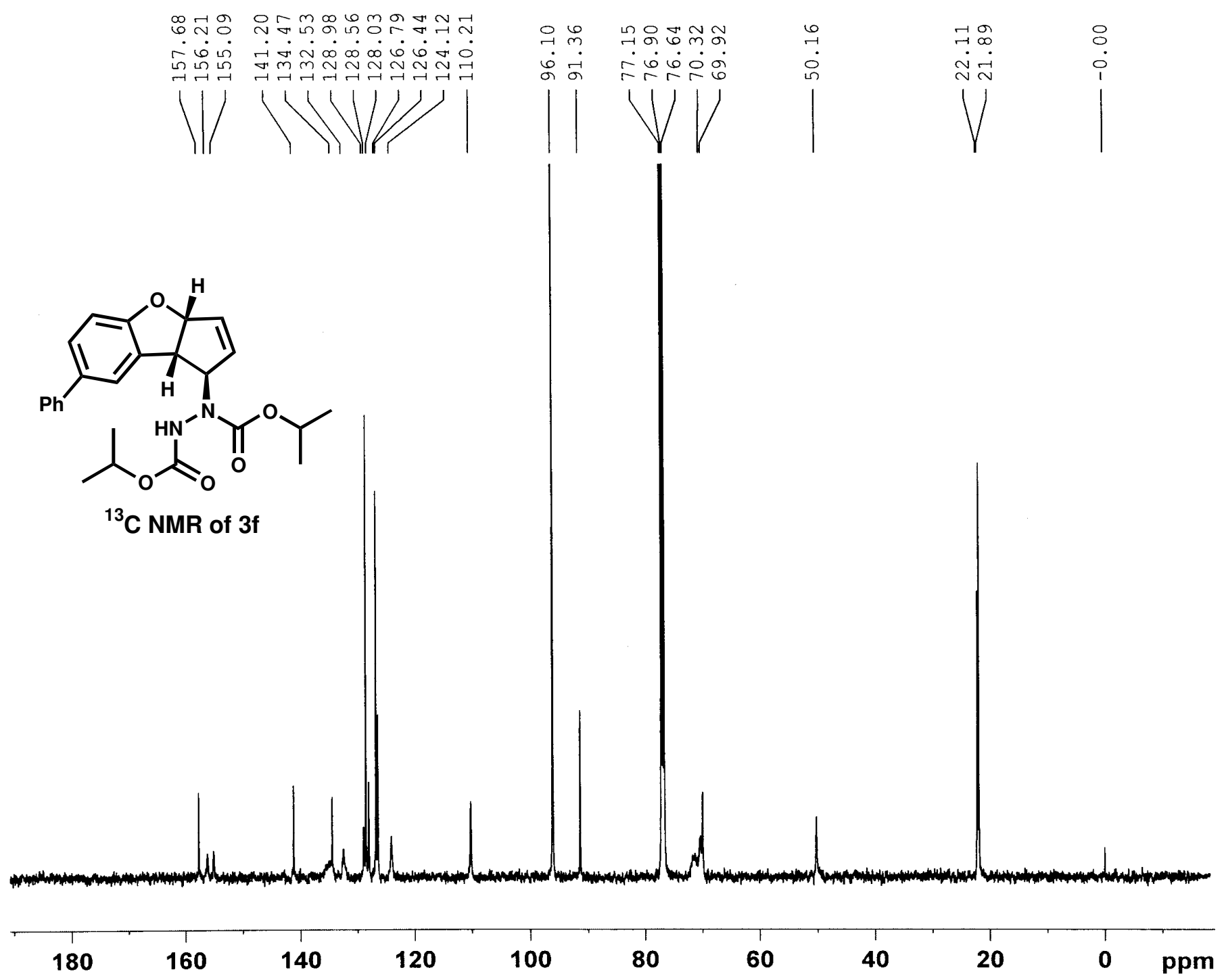



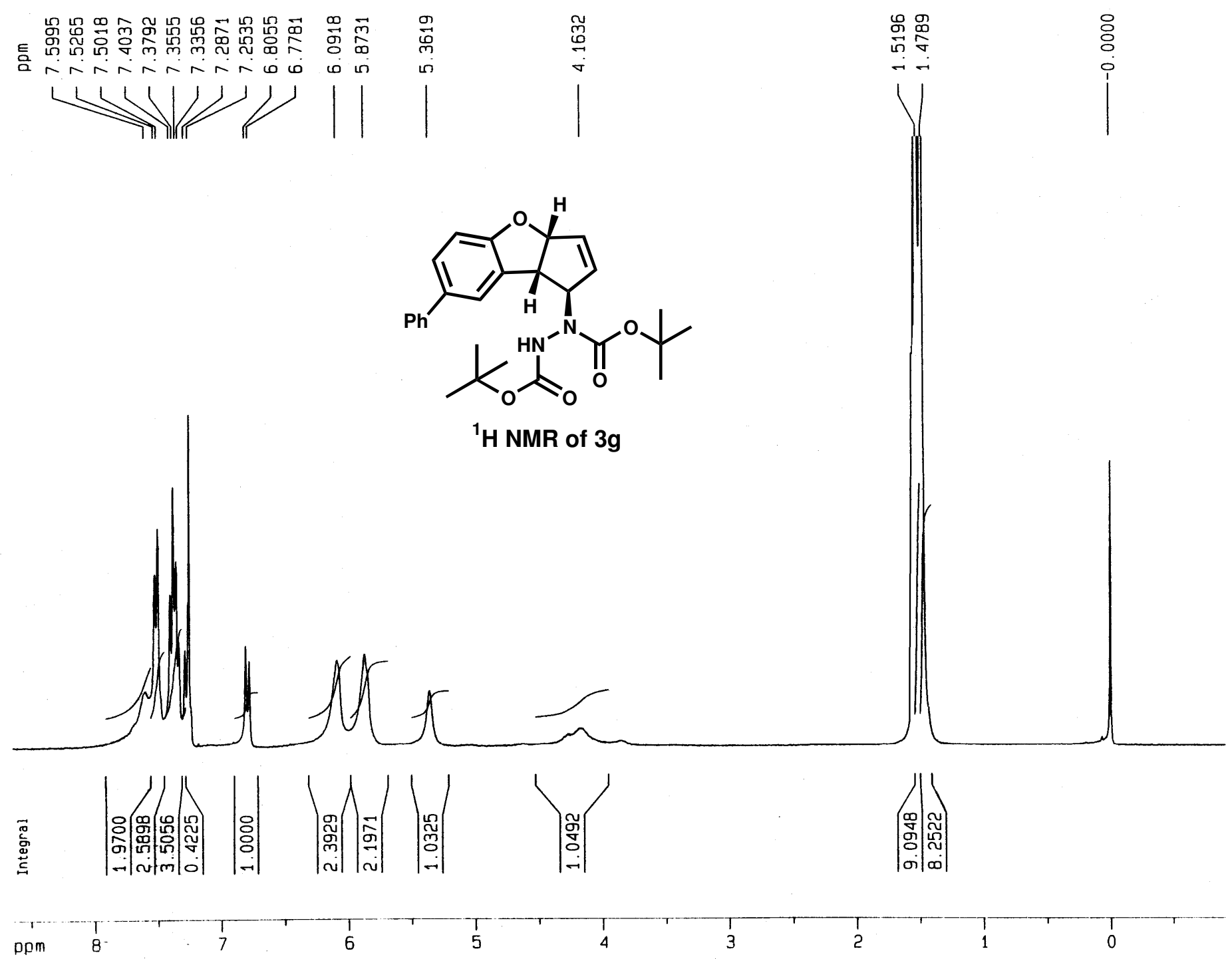


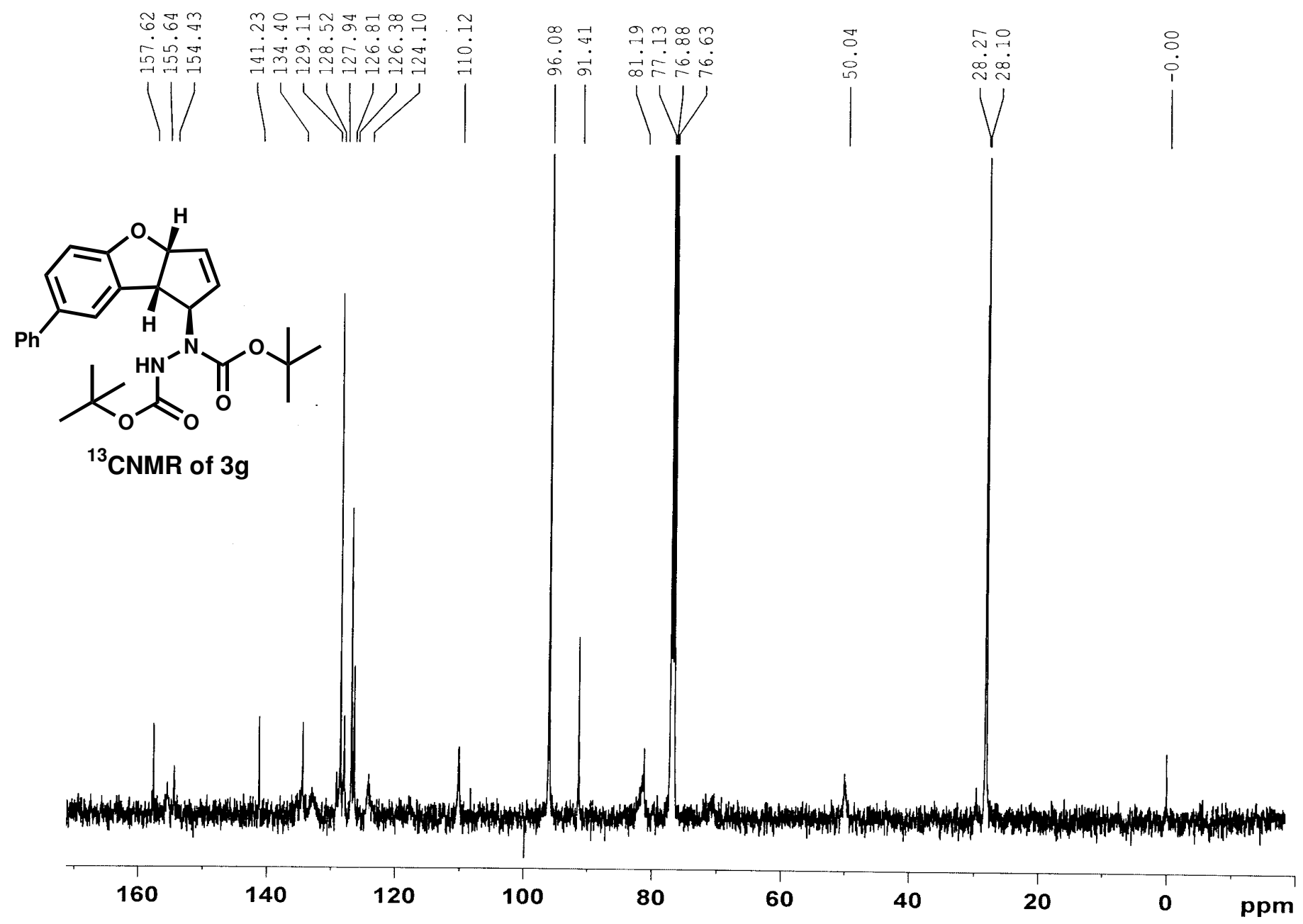



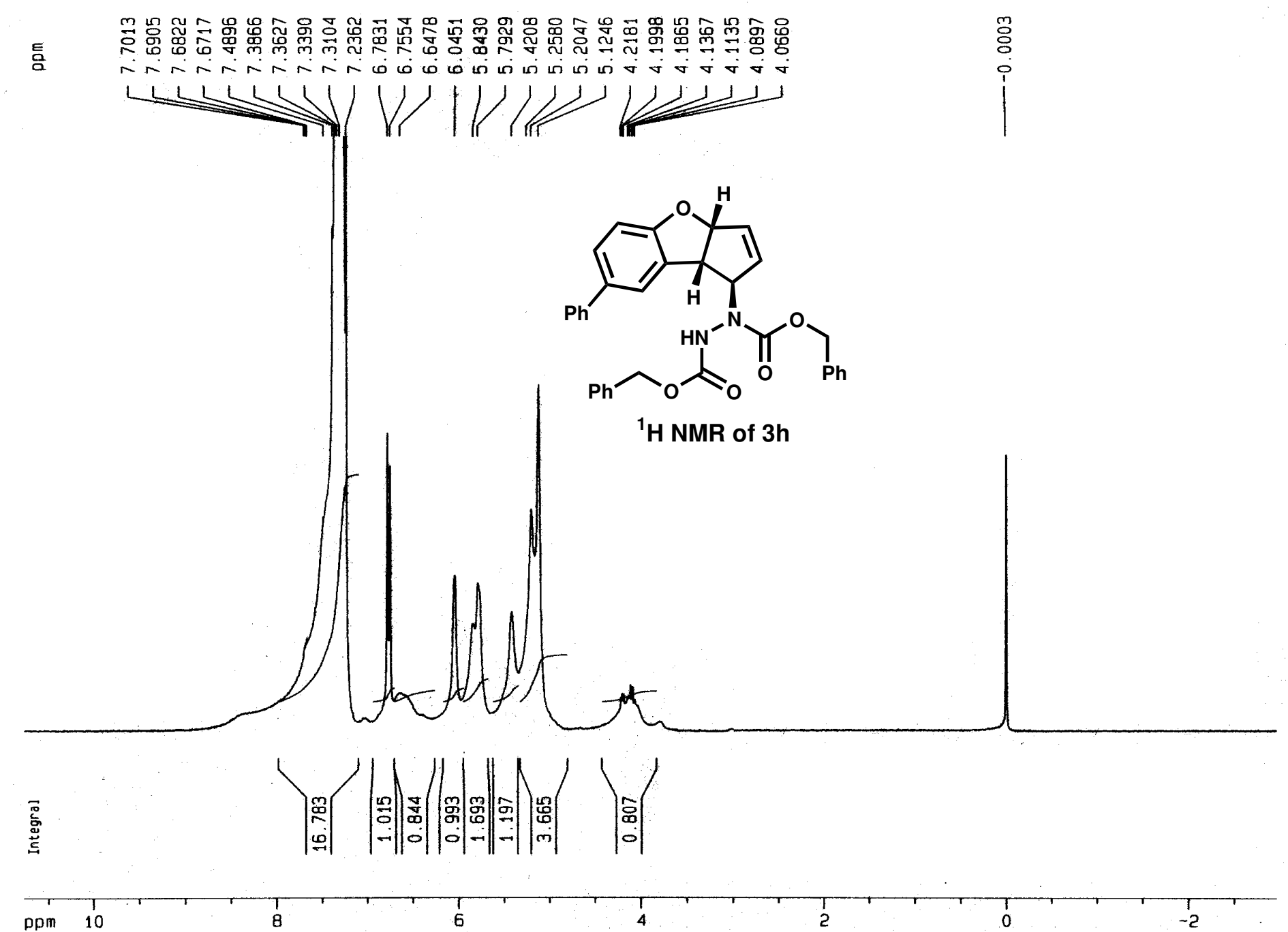


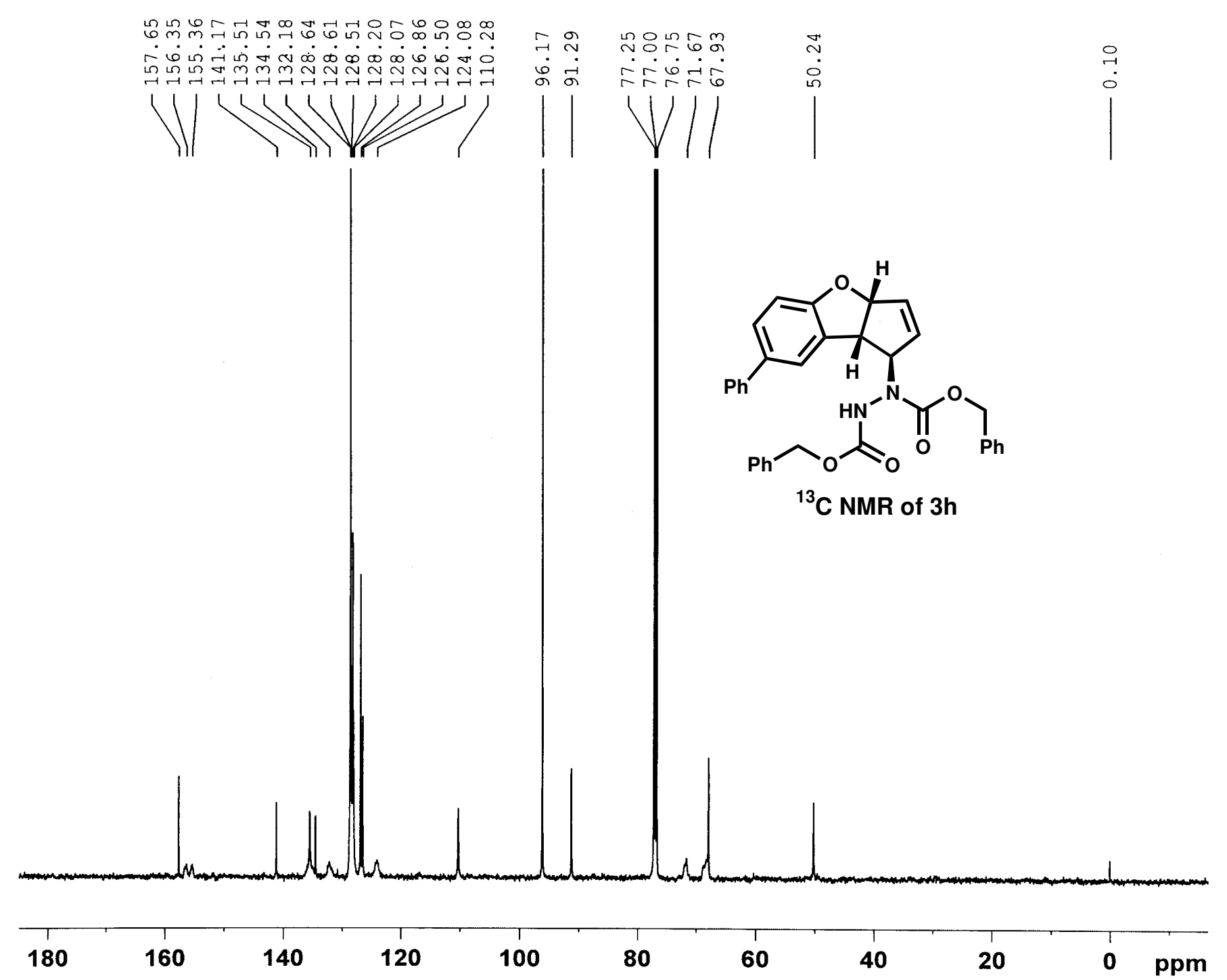




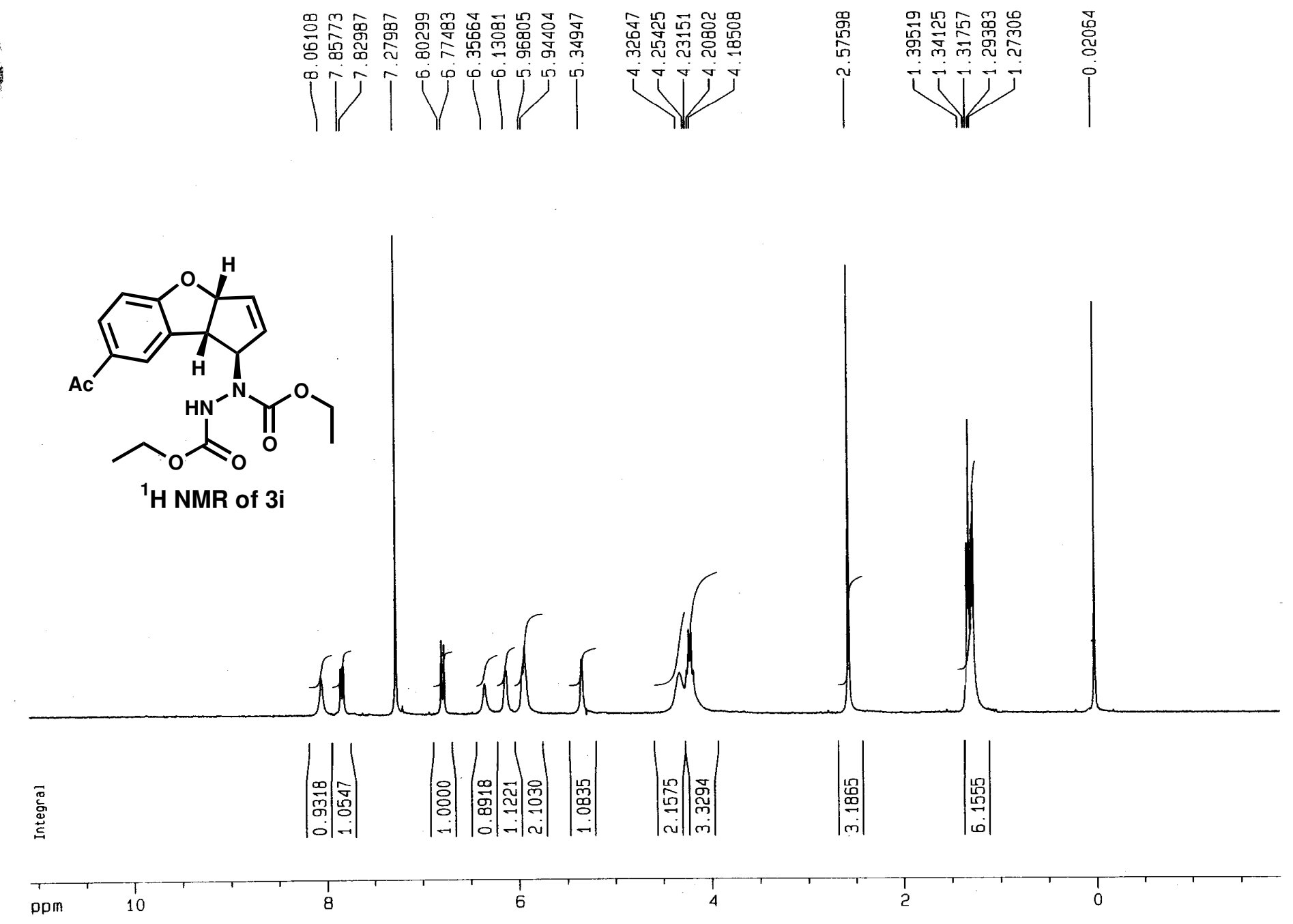




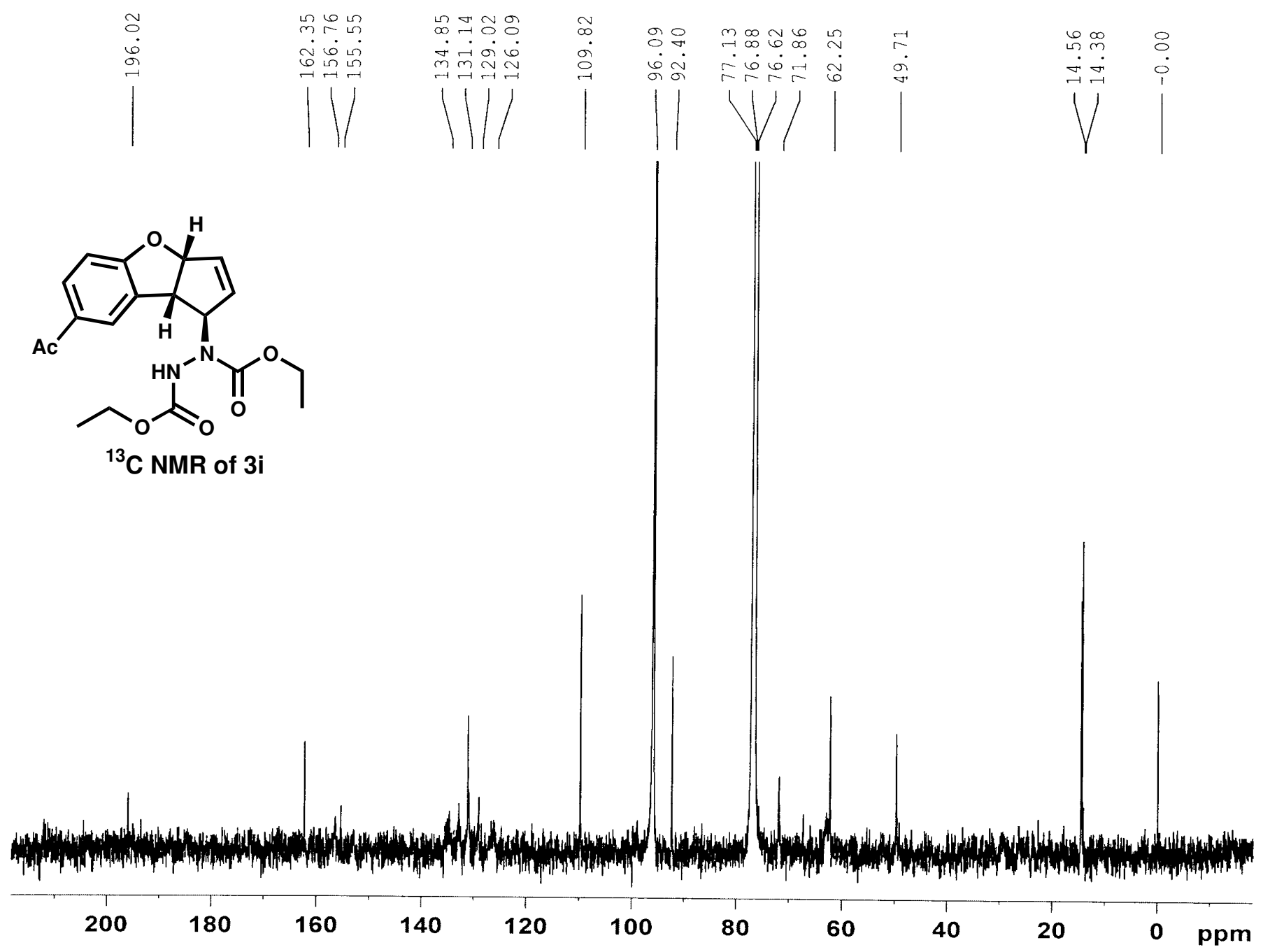




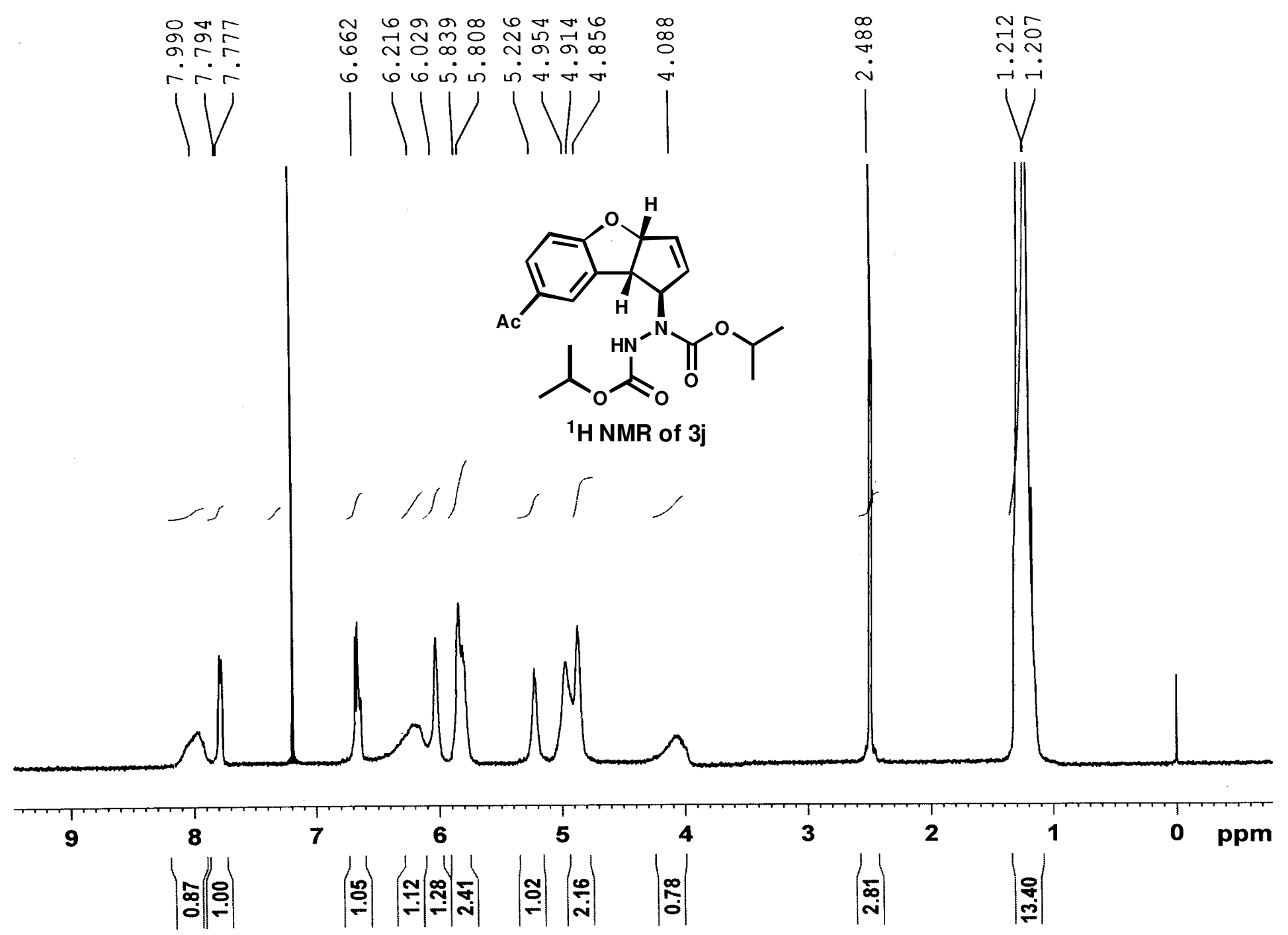




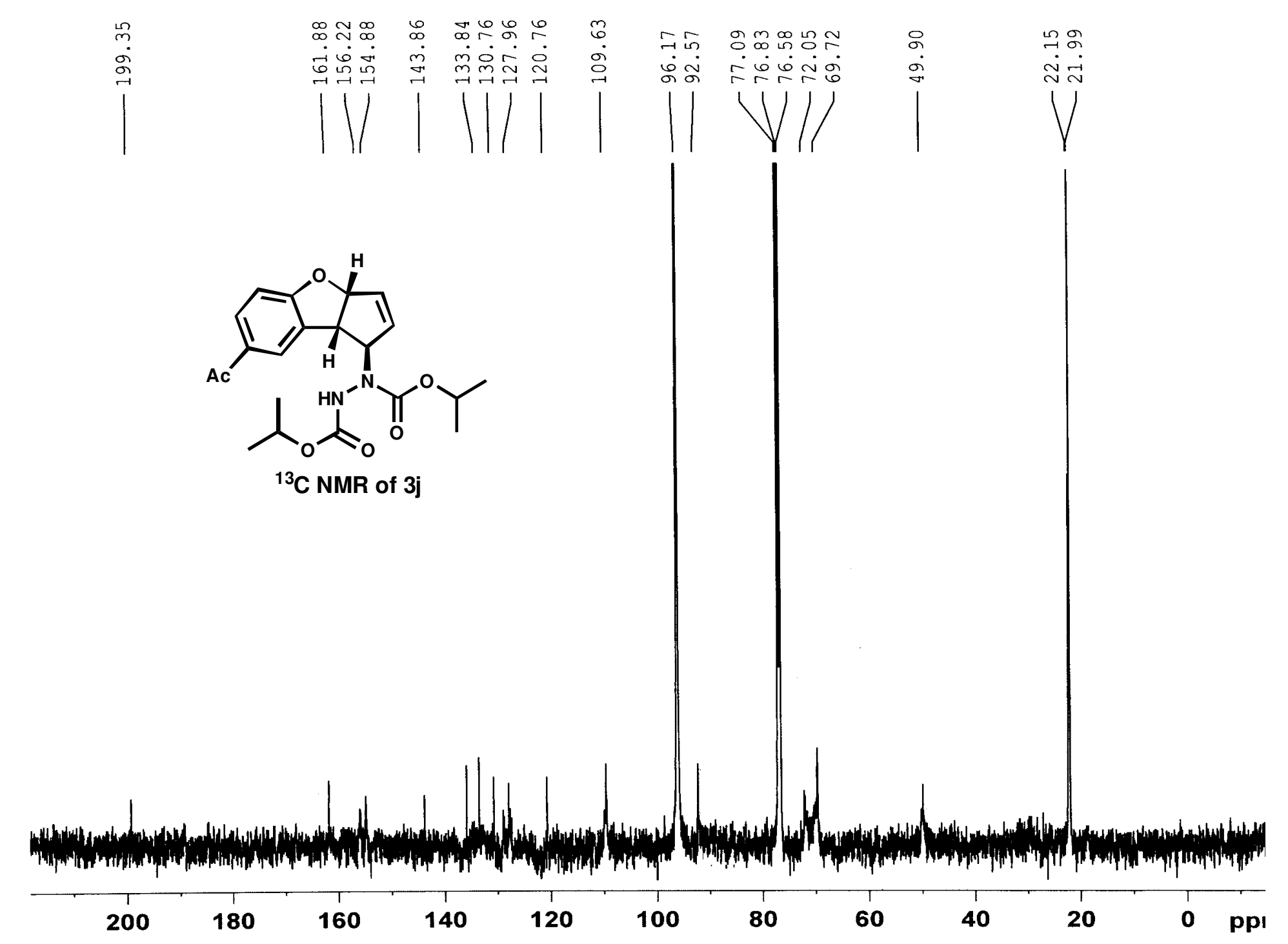




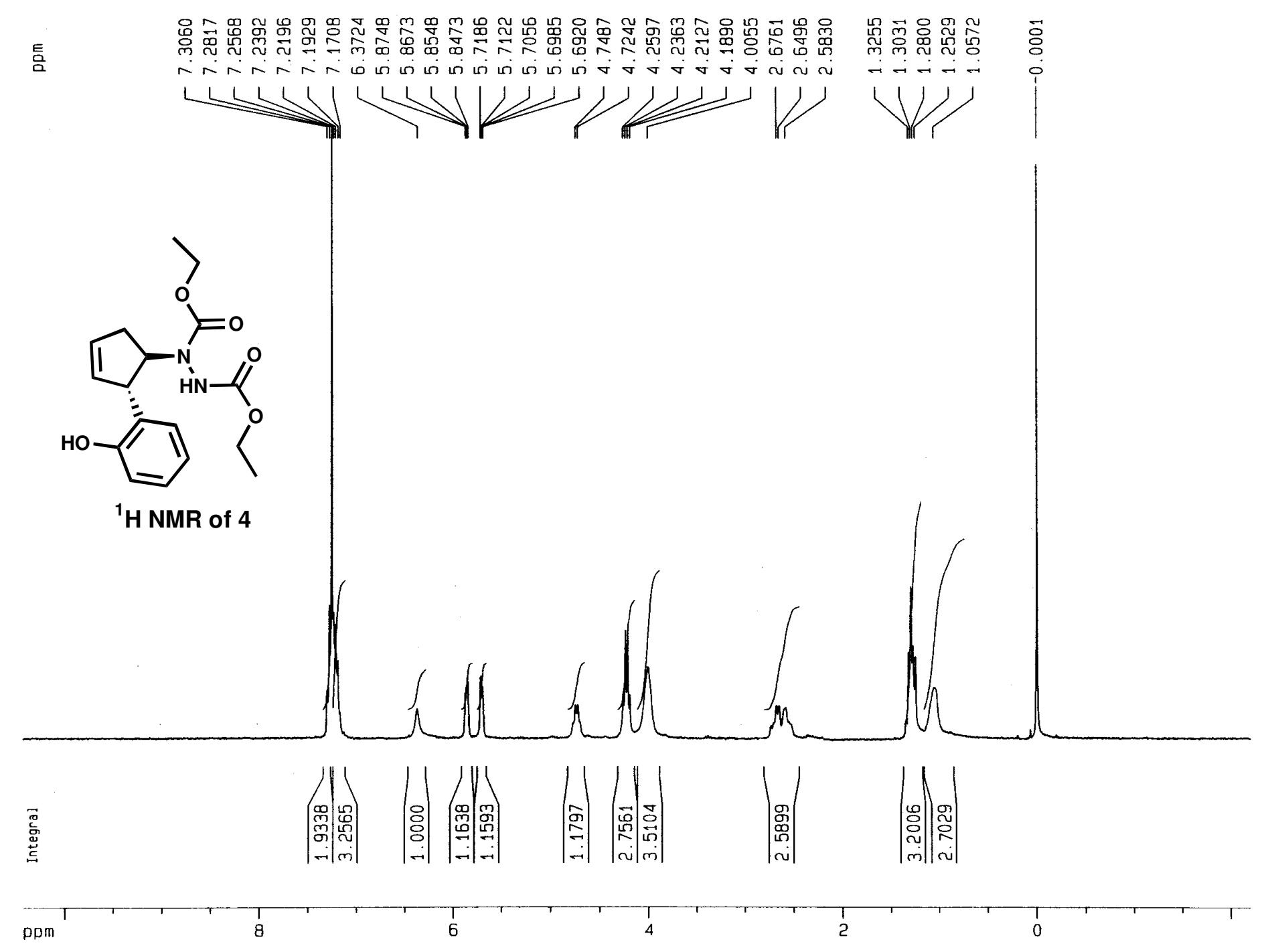




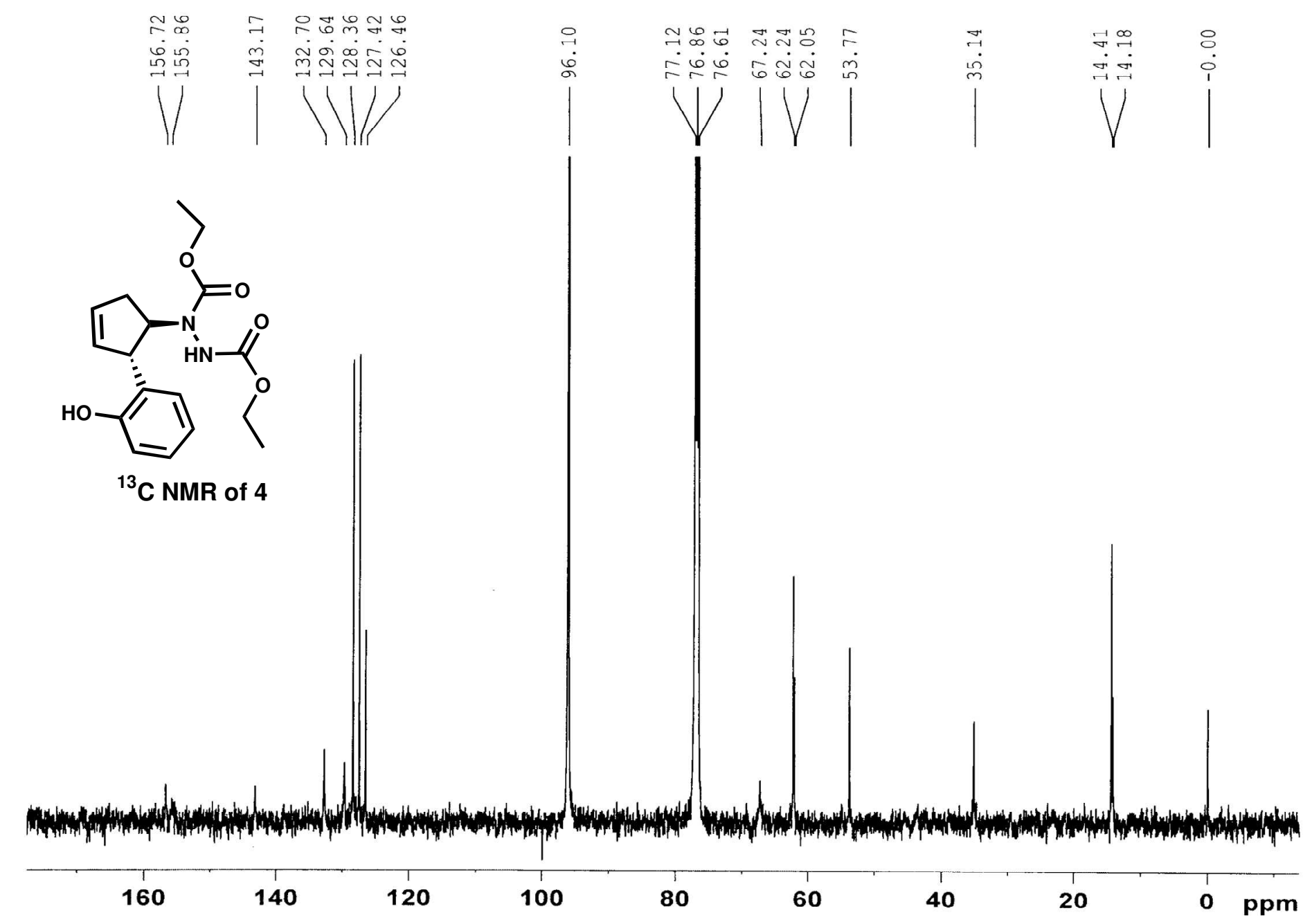




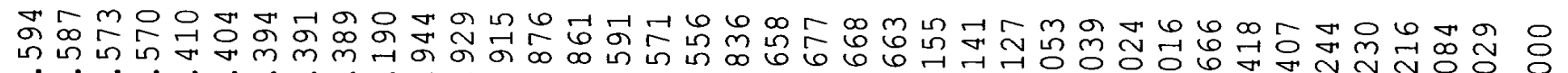

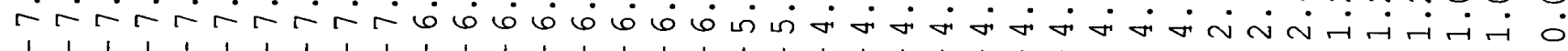
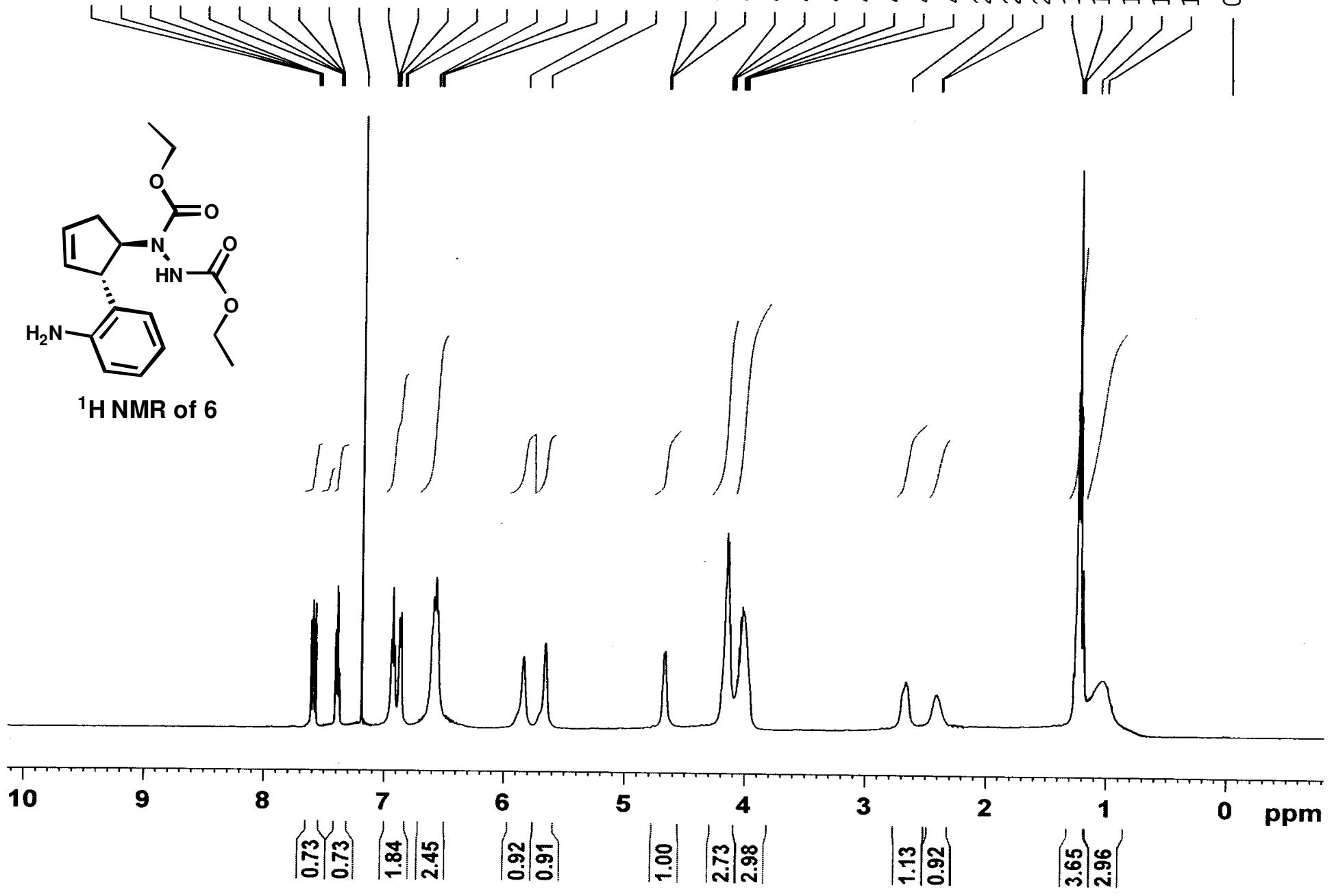


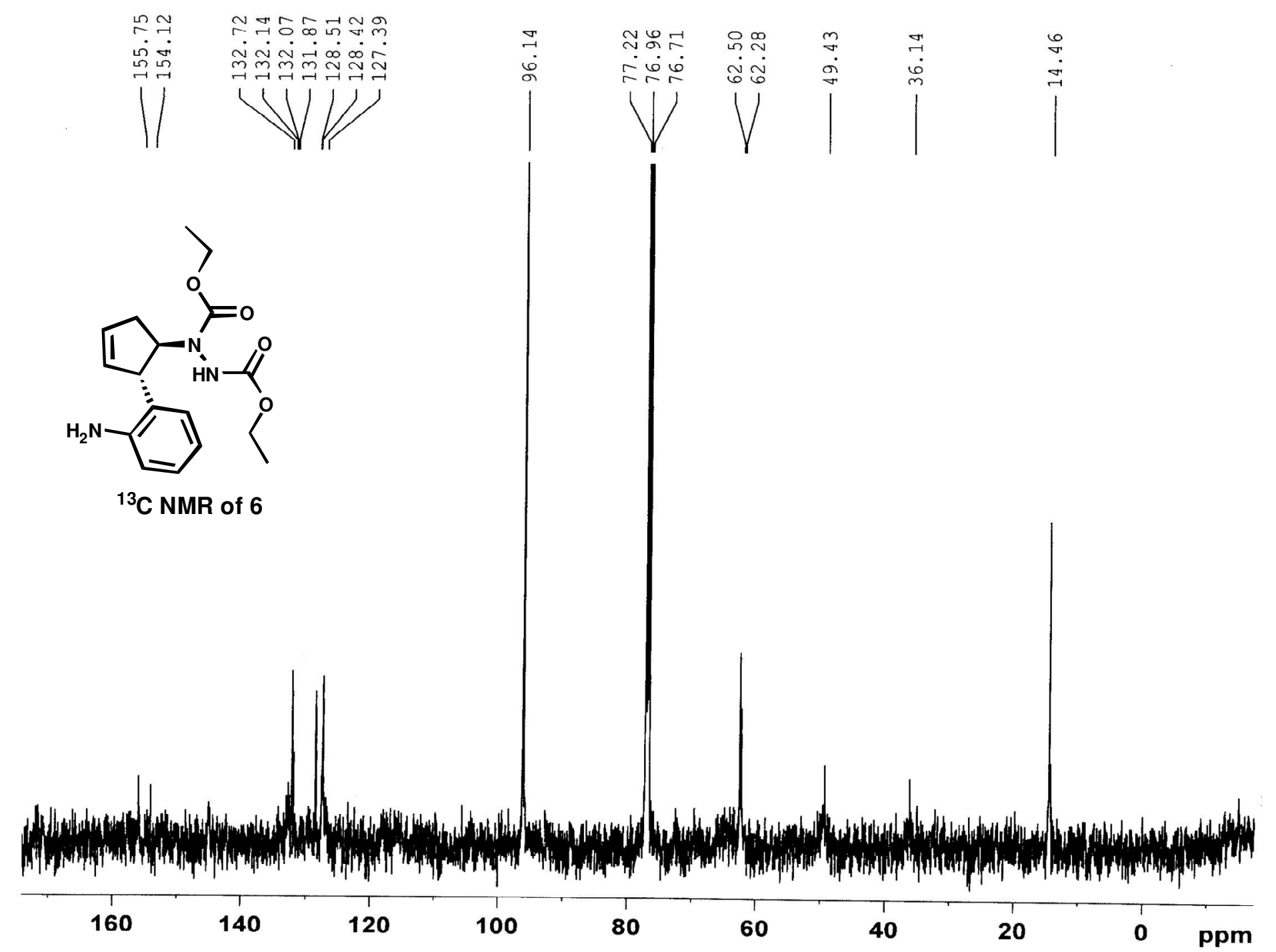




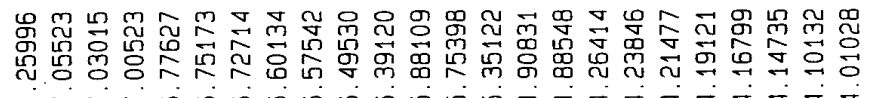

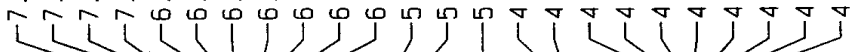

$\rightarrow$ tो

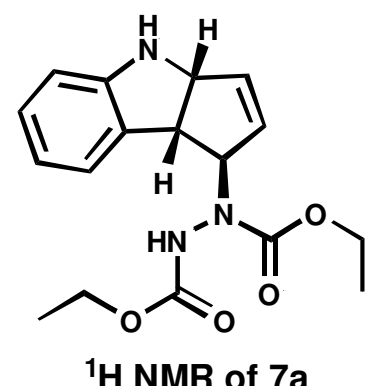

${ }^{1} \mathrm{H}$ NMR of $7 \mathrm{a}$

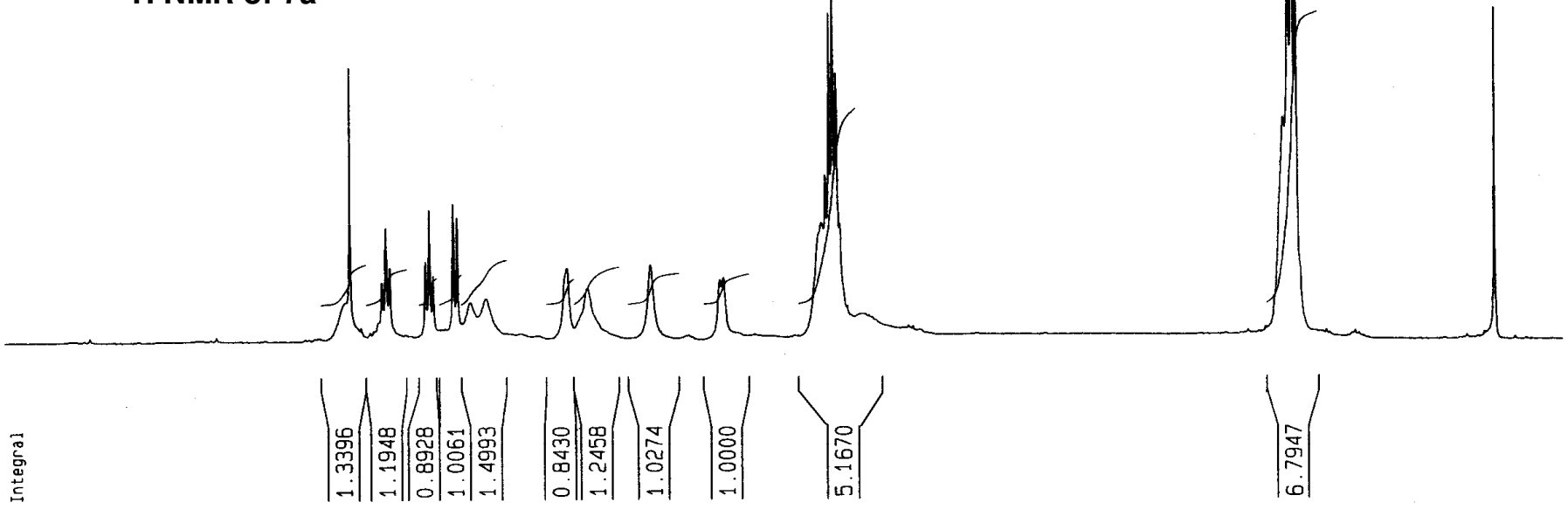

ppm

6

5

3 


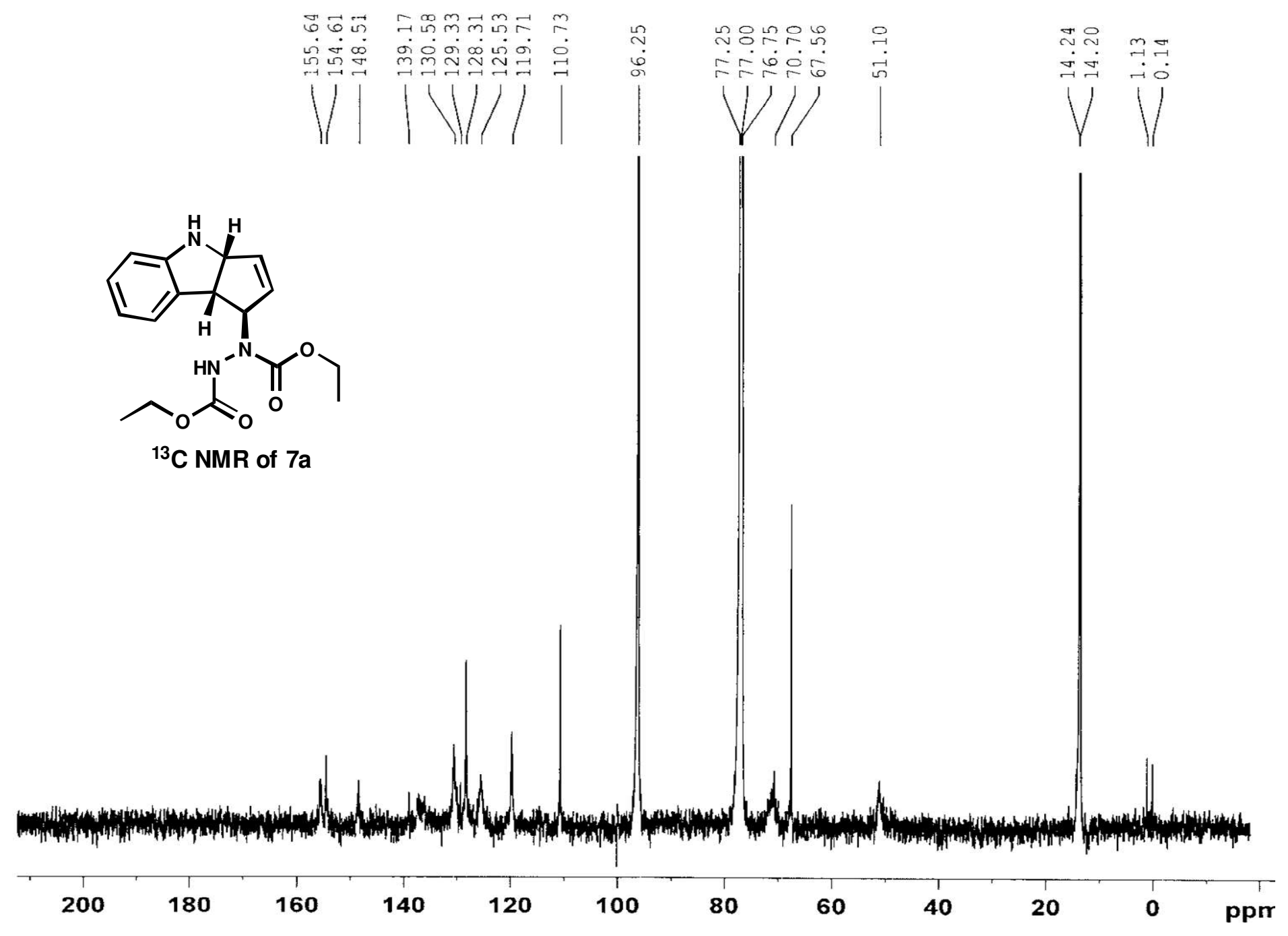




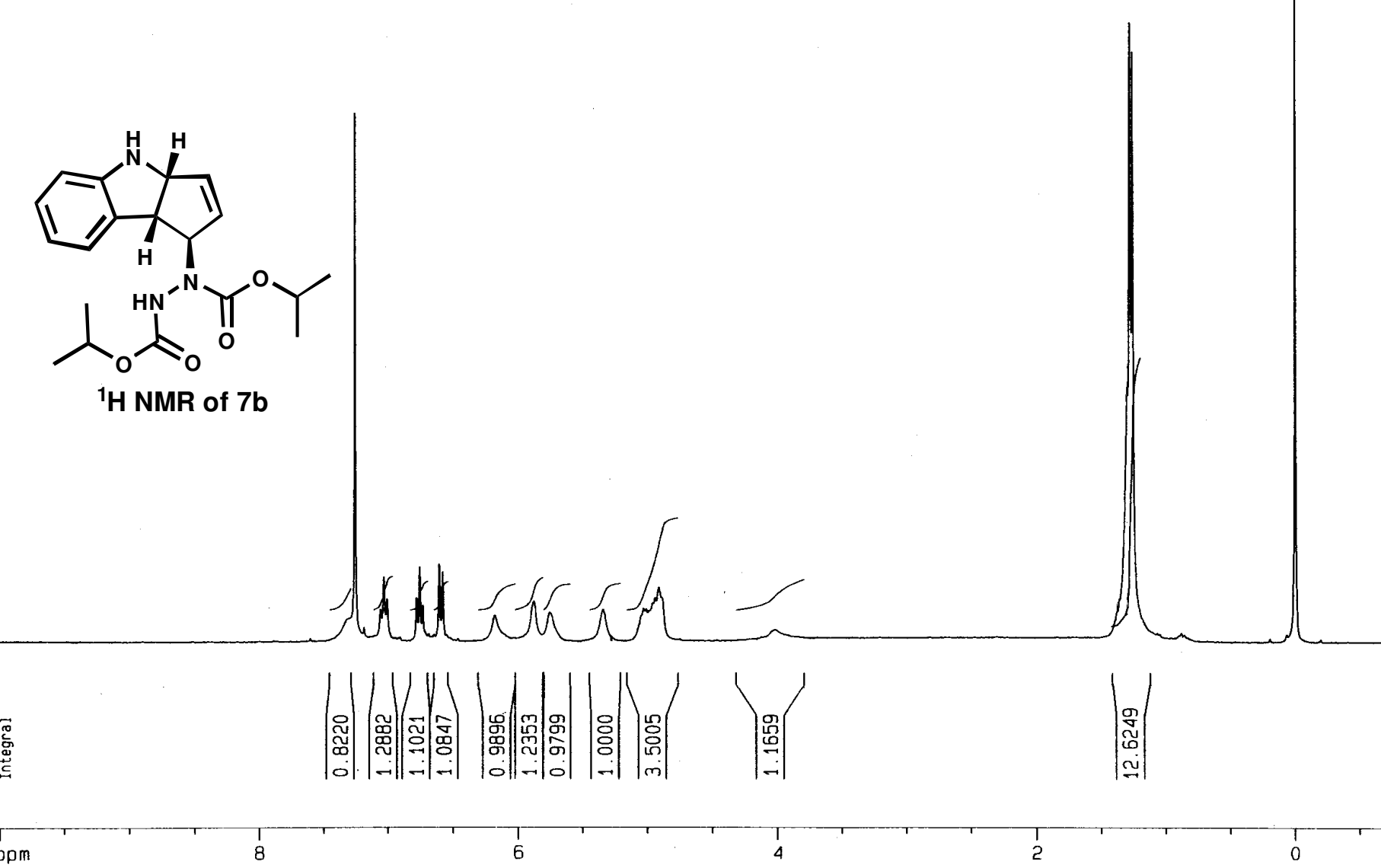




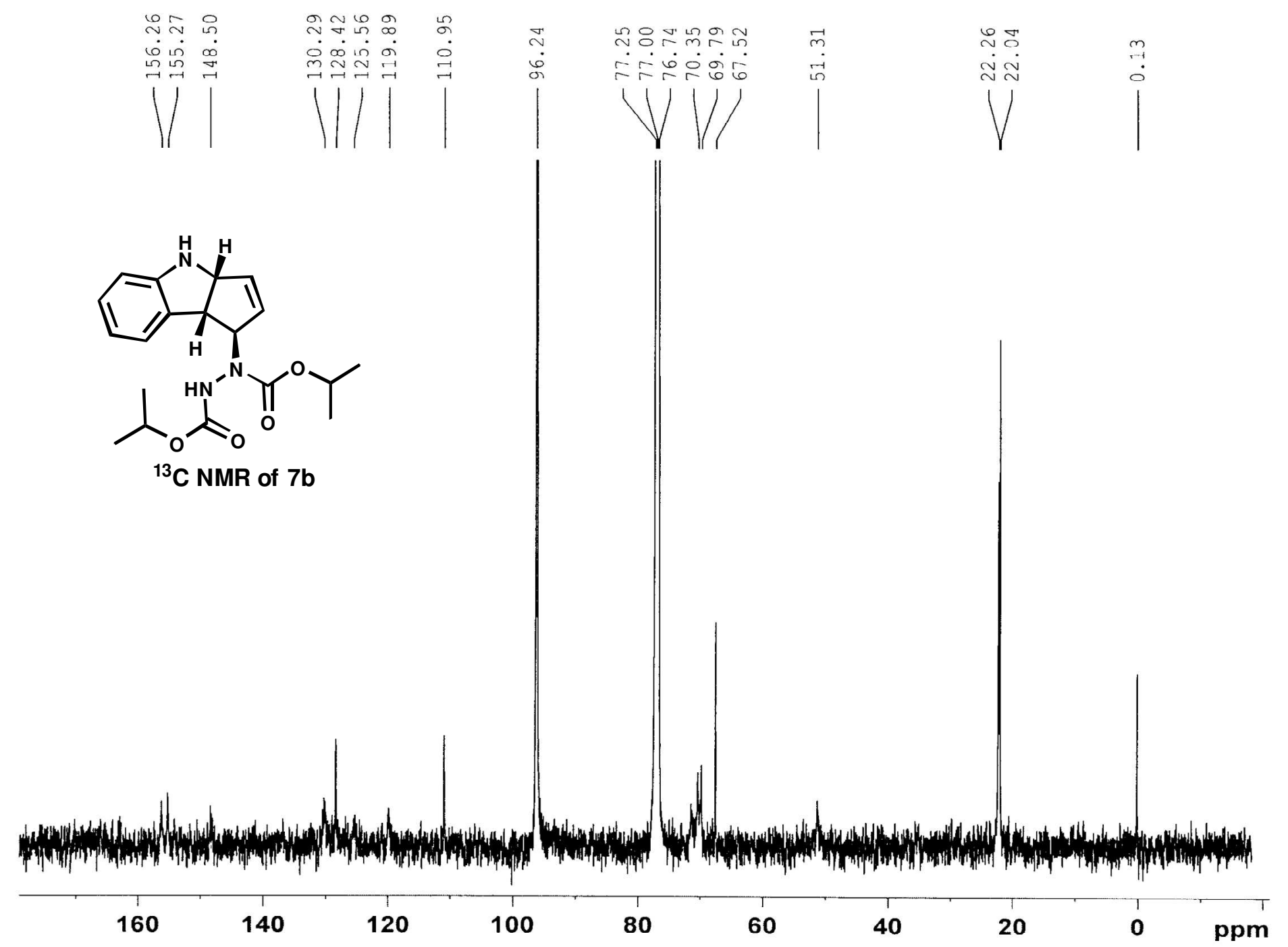




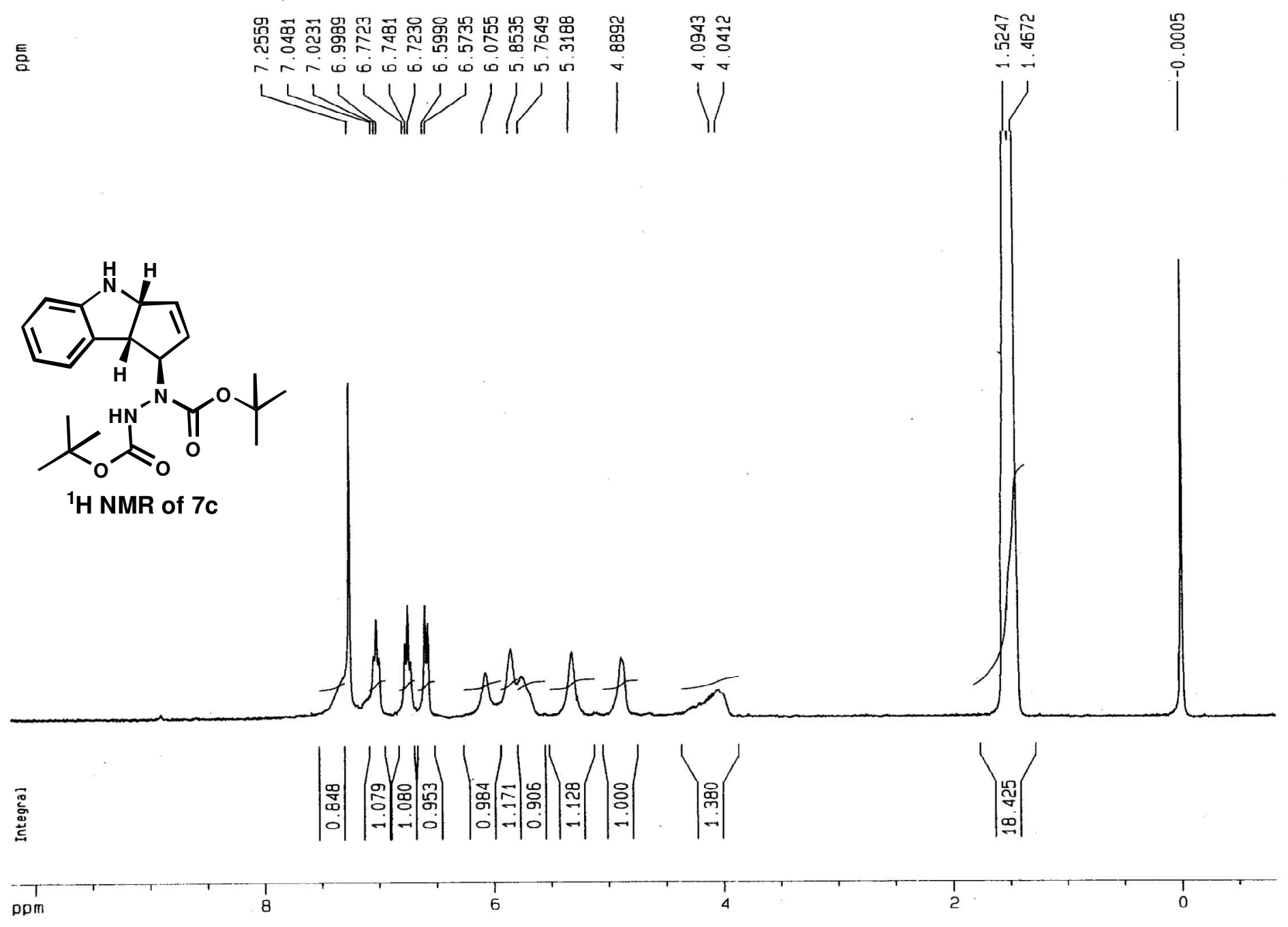




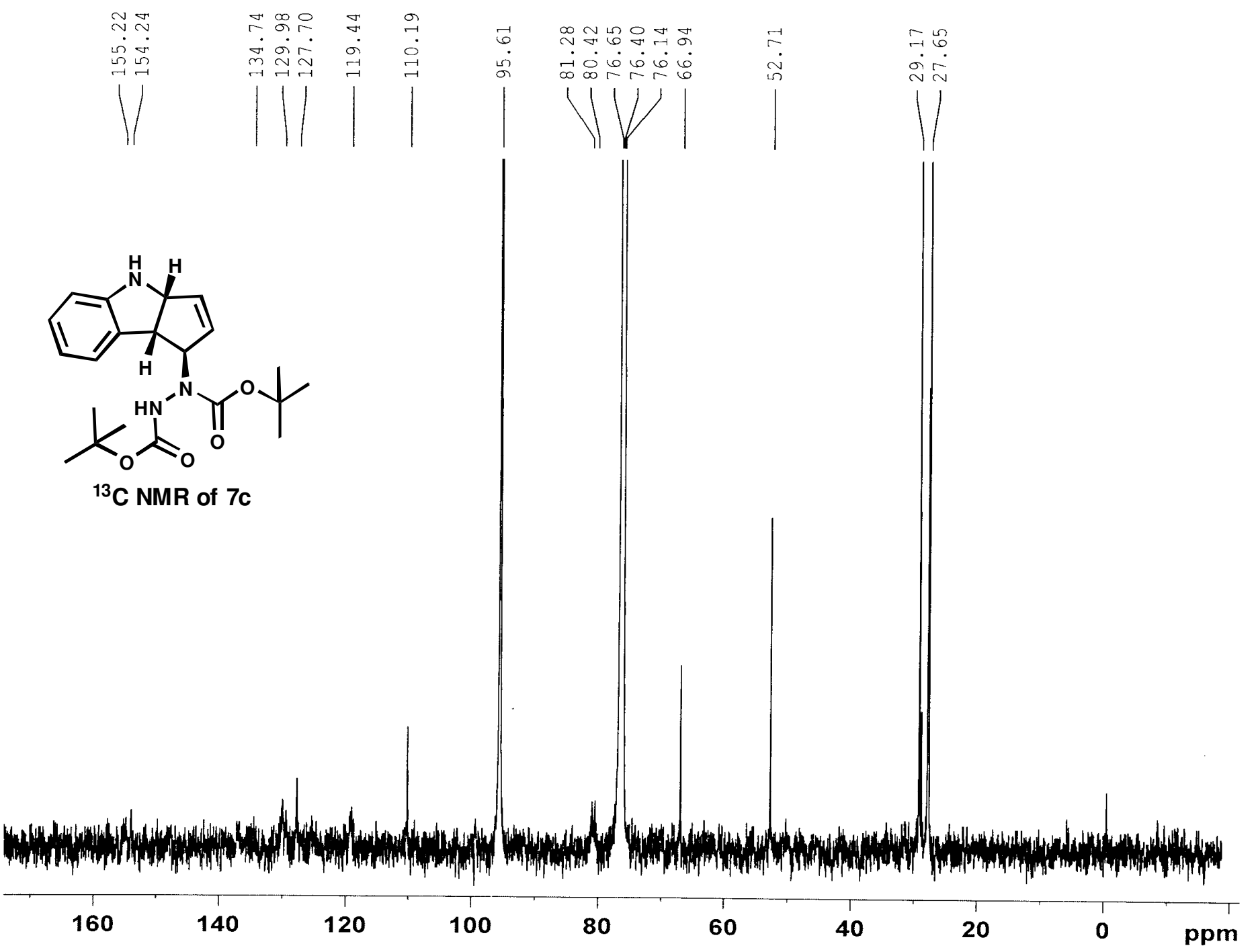

